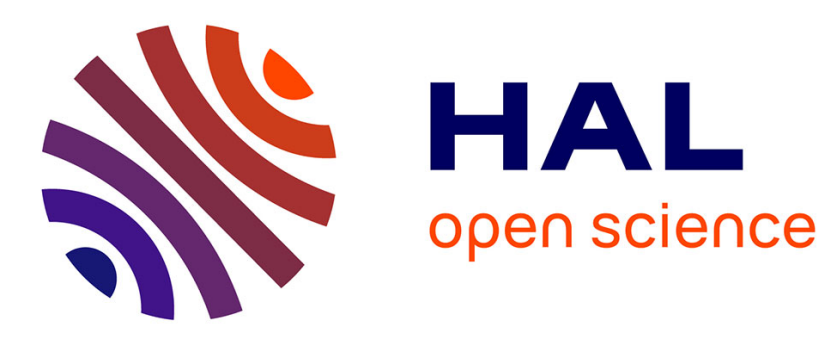

\title{
A time-incremental homogenization method for elasto-viscoplastic particulate composites based on a modified secant formulation
}

Stéphane Berbenni

\section{- To cite this version:}

Stéphane Berbenni. A time-incremental homogenization method for elasto-viscoplastic particulate composites based on a modified secant formulation. International Journal of Solids and Structures, 2021, pp.111136. 10.1016/j.ijsolstr.2021.111136 . hal-03278434

\section{HAL Id: hal-03278434 \\ https://hal.univ-lorraine.fr/hal-03278434}

Submitted on 5 Jul 2021

HAL is a multi-disciplinary open access archive for the deposit and dissemination of scientific research documents, whether they are published or not. The documents may come from teaching and research institutions in France or abroad, or from public or private research centers.
L'archive ouverte pluridisciplinaire HAL, est destinée au dépôt et à la diffusion de documents scientifiques de niveau recherche, publiés ou non, émanant des établissements d'enseignement et de recherche français ou étrangers, des laboratoires publics ou privés. 


\title{
A time-incremental homogenization method for elasto-viscoplastic particulate composites based on a modified secant formulation
}

\author{
Stéphane Berbenni a,b,1 \\ ${ }^{a}$ Université de Lorraine, Arts et Métiers Paris Tech, CNRS, LEM3, F-57000 \\ Metz, France \\ ${ }^{\mathrm{b}}$ Laboratory of Excellence on Design of Alloy Metals for low-mAss Structures \\ (DAMAS), Université de Lorraine, France
}

\begin{abstract}
Starting from the exact solution of the heterogeneous linear viscoelastic Eshelby ellipsoidal inclusion problem obtained in the time domain for an ellipsoidal inclusion embedded in an isotropic matrix (Berbenni et al., 2015), a direct time-incremental homogenization Mori-Tanaka scheme for two-phase non linear elasto-viscoplastic materials is proposed. The extension to non-linear behavior is based on a "modified secant" formulation to obtain the linear comparison composite (LCC) properties. In contrast with more classic homogenization method based on the first moment of stresses (i.e. classic secant formulation), the present approach is based on the secondorder moment of stresses making use of the Hill's lemma. Hence, a modified secant viscoplastic compliance is considered for the matrix phase. The effective behavior as well as the evolution laws of the averaged strains and stresses per phase are directly solved in the time domain, without the need of Laplace-Carson transforms. The estimates provided by the present homogenization model are compared to full-field
\end{abstract}


calculations from the literature for two-phase non linear particulate composites constituted of an isotropic elasto-viscoplastic matrix phase and isotropic elastic particles, and, for radial and non-radial loadings.

Keywords: Homogenization; Elasto-Viscoplastic material; Particulate Composites; Time-incremental method; Mori-Tanaka estimate; Modified Secant formulation

\section{Introduction}

Materials like composites or metallic alloys exhibit time-dependent behaviors, which are elasto-viscoplastic (or non linear viscoelastic) and involve different orders of time differentiation. This leads to a "long term memory effect" due to spatial-temporal interactions between the constituents of these composite materials. As an illustration of this effect, the effective (overall) behavior of an aggregate of Maxwellian constituents is no more Maxwellian (Suquet, 1987). In addition, such materials exhibit non-linear behaviors, which make more difficult the prediction accuracy of mean field homogenization models in comparison with full-field computations. Generally, mean field homogenization models first require a linearization step, which needs to be optimized, and, which is crucial to derive accurate estimates of the effective behavior and phase responses.

Two main homogenization strategies were reported in the literature for elastoviscoplastic composites and polycrystals. The first is based on Riemann-Stieltjes integral-based "hereditary" homogenization approaches for non ageing linear vis-

$\overline{1}$ Corresponding author. This paper is dedicated to Professor Henryk Petryk on the occasion of his 70th birthday.

Email address: stéphane.berbenni@univ-lorraine.fr (Stéphane Berbenni) 
coelastic materials using Laplace-Carson (LC) transforms and the correspondence principle (Mandel, 1966). Different homogenization schemes were developed using this principle (Hashin, 1969; Laws and McLaughlin, 1978; Wang and Weng, 1992; Weng, 1993; Turner and Tomé, 1993; Rougier et al., 1993, 1994; Li and Weng, 1994, 1997; Brinson and Lin, 1998; DeBotton and Tevet-Deree, 2004; Pierard and Doghri, 2006; Masson and Zaoui, 1999). However, LC inversion is needed at each resolution step in these homogenization methods, which severely limits their practical applications.

The second more efficient way is based on time-incremental "internal variables approaches", whereby the stress history in phases is recorded through internal variables. The advantage is to directly provide the effective behavior of the composite in the time domain avoiding LC inversions. Besides, complex loading paths in time domains and ageing behaviors can be treated. Different formulations were developed these last decades in this direction. In particular, the "additive interaction" (AI) law for the Maxwellian Eshelby inclusion problem was derived by Kouddane et al. (1993); Molinari et al. (1997); Molinari (2002); Mercier et al. (2005), and, more recently applied to the Mori-Tanaka (MT) and self-consistent (SC) estimates, using a "tangent" linearization formulation in Mercier and Molinari (2009); Mercier et al. (2012). The numerical results of the AI for non-linear particulate elasto-viscoplastic composites were compared to full-field finite element (FEM) calculations in Czarnota et al. (2015). In the context of polycrystal plasticity, different extensions of the AI law were developed by Wang et al. (2010), and, more recently by Zecevic and Lebensohn (2020). The "translated fields" (TF) method was developed for linear viscoelastic and elasto-viscoplastic composites in Paquin et al. (1999) and in Sabar et al. (2002) using a classic "secant" formulation. The TF method was adapted to treat linear viscoelastic composites with the MT scheme in Mercier et al. (2012), where it was successively compared to the AI law and full-field Fast Fourier Transform (FFT) cal- 
culations. The TF method was extended to non linear elasto-viscoplastic composites and polycrystals with a first order "affine" formulation to improve the effective behavior estimates of composites (Berbenni and Capolungo, 2015) and polycrystals (Mareau and Berbenni, 2015; Lhadi et al., 2018). Another original approach was proposed by Kowalczyk-Gajewska and Petryk (2011), in which the linearized subproblems for an inhomogeneity/matrix system with elastic or viscoplastic interaction rules were solved sequentially within one incremental step. This approach was developed for both MT and SC homogenization schemes. Doghri et al. (2010) developed an incremental-homogenization model based on a first order "affine" linearization and a fully implicit backward Euler scheme with an algorithmic tangent operator. Their numerically efficient method was applied to inclusion-reinforced elasto-viscoplastic composites for non-monotonic and non-proportional loadings and were compared to FEM calculations. In Wu et al. (2017), an incremental-secant homogenization model using an isotropic "modified secant" formulation was derived making use of the second moment of stresses. An "internal variables" approach inspired from the collocation method as used in LC methods was developed by Ricaud and Masson (2009) for two-phase composites, which gives exact and approximate results for the MT and the SC estimates, respectively. The extension of the this method to the case of linear anisotropic polycrystals with a SC procedure was reported in $\mathrm{Vu}$ et al. (2005) and in Masson et al. (2012). More recently, this approach was also extended to non-linear elasto-viscoplastic particulate composites with ageing effects in Masson et al. (2020), using a "modified secant" formulation as linearization for the viscoplastic strain rate in the matrix phase. A recent "internal variables" approach was introduced in Berbenni et al. (2015) and Berbenni and Sabar (2017). It is based on an exact incremental Eshelby-based homogenization scheme for linear viscoelastic heterogeneous materials. In Berbenni et al. (2015), the exactness of the obtained stress interaction law was demonstrated for an arbitrary anisotropic and 
ellipsoidal inhomogeneity embedded in an infinite matrix with isotropic properties, in comparison with the corresponding exact result given by the LC formulation.

Based on an implicit Euler time discretization scheme, different variational approaches were first developed in linear viscoelasticity (Lahellec and Suquet, 2007a; Badulescu et al., 2015) and for elasto(visco)plastic materials by Lahellec and Suquet (2007b) and Brassard et al. (2012). Using this time discretization, the variational approach for elasto-viscoplastic composites is based on the estimates of mechanical fields at each time step, working with an optimal linear thermo-elastic comparison composite. The variational approach to solve this problem was further improved by Lahellec and Suquet (2013), using a condensed dissipation potential in a "Rate Variational Principle (RVP)", and by Agoras et al. (2016) using a condensed stress potential in an "Incremental Variational Procedure (IVP)". More recently, Cotelo et al. (2020) provided an approximate "differential" form of the IVP, which gave exact results, such as the MT estimates for isotropic incompressible linear viscoelastic composites as well as accurate estimates for non-linear elasto-viscoplastic particulate composites. The IVP was also successfully applied to estimate the macroscopic responses and field statistics of elasto-viscoplastic polycrystals in Das and Ponte Castañeda (2021).

Inspired by several recent studies on homogenization-based methods considering second moments of stresses in two-phase Maxwellian composites (Lahellec and Suquet, 2013; Badulescu et al., 2015; Masson et al., 2020), the objective of this work is to extend the recently developed internal variables approach of Berbenni et al. (2015) and Berbenni and Sabar (2017) to non-linear elasto-viscoplastic behaviors using a "modified secant" formulation in the framework of a homogenization MT scheme. The present homogenization model is called the "MT-SEC-MODIF" formulation. For single potential materials (in linear elasticity, viscoplasticity ...) and making use 
of a "Linear Comparison Composite" (LCC) (Ponte Castañeda, 1991), it was proved that the "modified secant" formulation significantly improves the estimates of the effective behavior of composites with large mechanical contrasts between phases, such as voided materials or particulate composites with rigid inclusions (Ponte Castañeda and Suquet, 1998; Qiu and Weng, 1991; Suquet, 1995; Hu, 1996). In contrast, the classical "secant" (SEC) formulation, which only considers the first order stress moments (i.e. the phase average stresses), tends to overestimate the effective behavior of such composites.

The paper is organized as follows. In section 2, one first recalls the main direct time-incremental equations for non linear elasto-viscoplastic composites. The integral equation is exactly solved by considering an isotropic homogeneous reference medium with isotropic elastic and viscoplastic properties (Berbenni et al., 2015). Starting from the exact solution of the heterogeneous linear viscoelastic Eshelby ellipsoidal inclusion problem obtained in the time domain for an ellipsoidal inclusion embedded in an isotropic elasto-viscoplastic matrix, a direct time-incremental homogenization Mori-Tanaka (MT) scheme for two-phase non linear elasto-viscoplastic materials is proposed in section 3. In section 4, the extension to non-linear behavior is presented, based on a "modified secant" formulation for the viscoplastic behavior of the matrix phase. In contrast with more classic homogenization method based on the first moment of stresses, the present approach is based on the second-order moment of stresses making use of the Hill's lemma (Hill, 1967). The applications of the present homogenization method based on the "MT-SEC-MODIF" formulation are reported for elasto-viscoplastic particulate composites reinforced by elastic particles in section 5. Some numerical comparisons with full-field calculations reported in Lahellec and Suquet (2013), Czarnota et al. (2015) and Masson et al. (2020) are discussed for different materials and loadings. 
In the whole paper, the Einstein's summation convention is used for repeated indices and the notations for a given quantity $X$ are the following:

- vector: $X$

- 2nd order tensor: $\underset{\sim}{X}$

- 4th order tensor: $\underset{\approx}{X}$

Their indicial notations are:

- vector: $X_{i}$

- 2nd order tensor: $X_{i j}$

- 4th order tensor: $X_{i j k l}$

The main symbols used are the following:

- The 2nd order unit tensor is $\underset{\sim}{\delta}$ where $\delta_{i j}=1$ if $i=j$ and $\delta_{i j}=0$ if $i \neq j$.

- The 4th order unit tensor is $\underset{\approx}{I}$ where $I_{i j k l}=\frac{1}{2}\left(\delta_{i k} \delta_{j l}+\delta_{i l} \delta_{k j}\right)$.

- The 4 th order projection tensor $\underset{\approx}{J}$ is given by $J_{i j k l}=\frac{1}{3} \delta_{i j} \delta_{k l}$.

- The 4th order projection tensor is $\underset{\approx}{K}$ is given by $K_{i j k l}=I_{i j k l}-J_{i j k l}=\frac{1}{2}\left(\delta_{i k} \delta_{j l}+\delta_{i l} \delta_{k j}\right)-$ $\frac{1}{3} \delta_{i j} \delta_{k l}$.

- "." deals with the simple product.

- ":" deals with the contracted product.

- " $\otimes "$ deals with the tensor product.

- The spatial convolution is denoted $\star$.

- The Eulerian temporal derivative of $\underset{\sim}{A}$ is denoted $\underset{\sim}{\dot{A}}$.

2 Time-incremental equations for non linear elasto-viscoplastic composites

A heterogeneous elasto-viscoplastic medium with volume $V$ and boundary $\partial V$ is considered with constituents characterized by linear elastic compliances $\underset{\approx}{\stackrel{s}{\sim} \text { and non }}$ 
linear viscoplastic strain rates $\underset{\sim}{\dot{\varepsilon}} v p=g(\underset{\sim}{\sigma})$, which are time and stress-dependent. The problem is treated within the framework of quasi-static equilibrium with infinitesimal strains and no volume forces. The non linear constitutive behavior for a phase is given by:

$$
\underset{\sim}{\dot{\varepsilon}}=\underset{\sim}{s}: \underset{\sim}{\dot{\sigma}}+\underset{\sim}{g}(\sigma)
$$

where $\underset{\sim}{\dot{\varepsilon}}$ is the total (compatible) strain rate, $\underset{\sim}{\sigma}$ is the equilibrated Cauchy stress. The viscoplastic strain rate $\left.\underset{\sim}{\dot{\varepsilon}^{v p}}=\underset{\sim}{g} \underset{\sim}{\sigma}\right)$ is a non linear function dependent on the stress tensor $\underset{\sim}{\sigma}$. The solution consists in determining the strain and stress rates satisfying all the field equations. In the following, in order to be able to apply analytical homogenization methods, this non linear behavior is approximated by a "Linear Comparison Composite" (LCC)(Ponte Castañeda, 1991). Therefore, the first step is to solve a linear viscoelastic Maxwellian problem with a linearized viscoplastic strain rate:

$$
\underset{\sim}{\dot{\varepsilon}}=\underset{\approx}{s}: \underset{\sim}{\dot{\sigma}}+\underset{\approx}{m}: \underset{\sim}{\sigma}
$$

where $\underset{\approx}{m}$ is the linearized viscoplastic compliance. In this paper, a "modified secant" formulation will be considered, see section 4 .

Due to the material's property heterogeneities, spatial fluctuations $\underset{\approx}{\delta}$ and $\underset{\approx}{\delta m}$ are defined with respect to an infinite medium with homogeneous properties $\underset{\approx}{s}$ and $\underset{\approx}{m^{0}}$ :

$$
\underset{\approx}{s}=\underset{\approx}{s^{0}}+\underset{\approx}{\delta s} ; \underset{\approx}{m}=\underset{\approx}{m^{0}}+\delta \underset{\approx}{m}
$$

where $\underset{\approx}{s}, \underset{\approx}{m^{0}}$ are linear elastic compliances and linearized viscoplastic compliances of the homogeneous reference medium. Using the Green's function technique and previous linearized constitutive equations for an applied homogeneous strain rate $\underset{\sim}{\dot{\varepsilon}^{0}}$ at the boundary $\partial V$ :

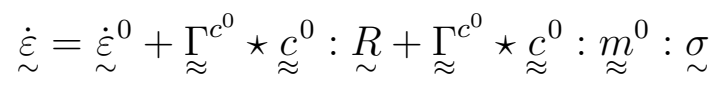

where $\star$ denotes spatial convolution, $\underset{\approx}{\Gamma^{0}}$ is the modified Green's tensor associated 
with $\underset{\approx}{c^{0}}$ defined as (Kröner, 1990):

$$
\Gamma_{i j k l}^{c^{0}}=-\frac{1}{2}\left(G_{i k, j l}^{c^{0}}+G_{j k, i l}^{c^{0}}\right)
$$

with $\underset{\sim}{G^{0}}$ the elastic Green's tensor (Mura, 1987). In Eq. 4, $\underset{\sim}{R}$ contains the fluctuations of both elastic and linearized viscoplastic compliances and is given by:

$$
\underset{\sim}{R}=\underset{\approx}{\delta}: \underset{\sim}{\dot{\sigma}}+\delta \underset{\sim}{m}: \underset{\sim}{\sigma}
$$

For the rest of the paper, one assumes the case of a homogeneous reference medium with isotropic elastic and anelastic (viscous) properties, where $k^{0}, k_{v}{ }^{0}$ are the elastic and anelastic (viscous) bulk moduli of the reference medium, and, $\mu^{0}, \mu_{v}{ }^{0}$ are its elastic and anelastic (viscous) shear moduli, such that:

$$
\stackrel{c}{0}^{0}=3 k^{0} \underset{\approx}{J}+2 \mu^{0} \underset{\approx}{K}
$$

and:

$$
\underset{\approx}{m^{0}}=\frac{1}{3 k_{v}^{0}} \underset{\approx}{J}+\frac{1}{2 \mu_{v}^{0}} \underset{\approx}{K}
$$

Then, using the identities $\underset{\approx}{J}: \underset{\sim}{\sigma}=\sigma_{m} \underset{\sim}{\delta}$ and $\underset{\approx}{K}: \underset{\sim}{\sigma}=\underset{\sim}{\sigma}-\sigma_{m} \underset{\sim}{\delta}$ with $\sigma_{m}$ the hydrostatic pressure $\left(\sigma_{m}=\frac{1}{3} \sigma_{k k}\right)$ :

$$
\underset{\approx}{c}: \underset{\approx}{m^{0}}: \underset{\sim}{\sigma}=\left(\frac{k^{0}}{k_{v}^{0}}-\frac{\mu^{0}}{\mu_{v}^{0}}\right) \sigma_{m} \underset{\sim}{\delta}+\frac{\mu^{0}}{\mu_{v}^{0}} \underset{\sim}{\sim}
$$

In this case, the elastic Green's tensor used in Eq. 5 reads (Walpole, 1966; Mura, 1987):

$$
G_{i j}^{c^{0}}\left(\underline{x}-\underline{x}^{\prime}\right)=\frac{1}{4 \pi \mu^{0}} \frac{\delta_{i j}}{\left|\underline{x}-\underline{x}^{\prime}\right|}-\frac{1}{8 \pi \mu^{0}} \frac{3 k^{0}+\mu^{0}}{3 k^{0}+4 \mu^{0}}\left|\underline{x}-\underline{x}^{\prime}\right|_{, i j}
$$

where $\underline{x}$ and $\underline{x}^{\prime}$ are position vectors.

The stress field $\underset{\sim}{\sigma}$ is statically admissible, therefore: $\underset{\approx}{{ }^{c}}{ }^{0} \star \underset{\sim}{\sigma}=0$, and, using Eq. 9:

$$
\left({\underset{\approx}{\Gamma}}^{c^{0}} \star \underset{\approx}{c^{0}}:{\underset{\approx}{m^{0}}}^{0} \underset{\sim}{\sigma}\right)_{i j}=\left(\frac{k^{0}}{k_{v}^{0}}-\frac{\mu^{0}}{\mu_{v}^{0}}\right) \int_{V} \Gamma_{i j k k}^{c^{0}}\left(\underline{x}-\underline{x}^{\prime}\right) \sigma_{m}\left(\underline{x}^{\prime}\right) d V^{\prime}
$$


From Eq. 5, Eq. 10, and the identity: $\left|\underline{x}-\underline{x}^{\prime}\right|_{, k k}=2\left|\underline{x}-\underline{x}^{\prime}\right|^{-1}$ :

$$
\Gamma_{i j k k}^{c^{0}}\left(\underline{x}-\underline{x}^{\prime}\right)=-\frac{1}{4 \pi} \frac{3}{3 k^{0}+4 \mu^{0}}\left(\frac{1}{\left|\underline{x}-\underline{x}^{\prime}\right|}\right)_{, i j}
$$

Therefore, Eq. 4 writes:

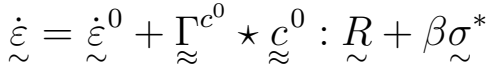

$$
\begin{aligned}
& \sigma_{i j}^{*}(\underline{x})=-\frac{1}{4 \pi} \int_{V}\left(\frac{1}{\left|\underline{x}-\underline{x}^{\prime}\right|}\right)_{, i j} \sigma_{m}\left(\underline{x}^{\prime}\right) d V^{\prime}
\end{aligned}
$$

where $\sigma_{i j}^{*}(\underline{x}) \rightarrow 0$ when $|\underline{x}| \rightarrow \infty$. Furthermore, from Eq. 11 and Eq. 12 together with Eq. 14, the constant $\beta$ is given by:

$$
\beta=\frac{3}{3 k^{0}+4 \mu^{0}}\left(\frac{k^{0}}{k_{v}^{0}}-\frac{\mu^{0}}{\mu_{v}^{0}}\right)
$$

Through Eq. 14, it is seen that $\underset{\sim}{\sigma^{*}}$ is a volume term, which is incrementally solved from its time derivative:

$$
\dot{\sigma}_{i j}^{*}(\underline{x})=-\frac{1}{4 \pi} \int_{V}\left(\frac{1}{\left|\underline{x}-\underline{x}^{\prime}\right|}\right)_{, i j} \dot{\sigma}_{m}\left(\underline{x}^{\prime}\right) d V^{\prime}
$$

Using Eqs. 2, 3, 6 together with Eq. 13 yields:

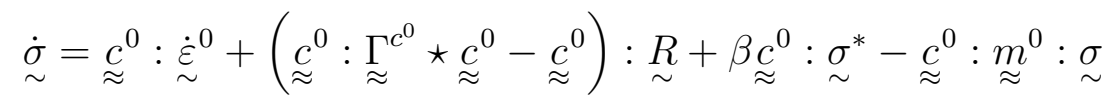

The rate of $\sigma_{m}(\underline{x})$ in Eq. 16 is obtained by taking the spherical part of Eq. 17 using Eq. 12:

$$
\dot{\sigma}_{m}(\underline{x})=3 k^{0} \dot{\varepsilon}_{m}^{0}-\frac{\alpha^{c^{0}}}{2 \pi} \int_{V}\left(\frac{1}{\left|\underline{x}-\underline{x}^{\prime}\right|}\right)_{, i j} R_{i j}\left(\underline{x}^{\prime}\right) d V^{\prime}-6 \alpha^{c^{0}} R_{m}(\underline{x})-\frac{\alpha^{c^{0}}}{\alpha^{b^{0}}} \sigma_{m}(\underline{x})
$$

where: 


$$
\begin{aligned}
\alpha^{c^{0}} & =\frac{3 k^{0} \mu^{0}}{3 k^{0}+4 \mu^{0}} \\
\alpha^{b^{0}} & =\frac{3 k_{v}^{0} \mu_{v}{ }^{0}}{3 k_{v}{ }^{0}+4 \mu_{v}{ }^{0}}
\end{aligned}
$$

Inserting Eq. 18 in Eq. 16 and after algebraic manipulations, the field $\underset{\sim}{\sigma}$ is obtained with the following time-differential equation already reported in Berbenni et al. (2015) and in Berbenni and Sabar (2017):

$\left.\dot{\sigma}_{i j}^{*}(\underline{x})=-\frac{\alpha^{c^{0}}}{4 \pi} \int_{V}\left|\underline{x}-\underline{x}^{\prime}\right|_{, i j k l} R_{k l}\left(\underline{x^{\prime}}\right)\right) d V^{\prime}+\frac{3 \alpha^{c^{0}}}{2 \pi} \int_{V}\left(\frac{1}{\left|\underline{x}-\underline{x^{\prime}}\right|}\right)_{, i j} R_{m}\left(\underline{x^{\prime}}\right) d V^{\prime}-\frac{\alpha^{c^{0}}}{\alpha^{b^{0}}} \sigma_{i j}^{*}(\underline{x})$

using $\int_{V}\left(\frac{1}{\left.\mid \underline{x-\underline{x}^{\prime} \mid}\right)_{, i j}} d V^{\prime}=0\right.$ and the following identity proved in Appendix A:

$$
\int_{V}\left(\frac{1}{\left|\underline{x}-\underline{x}^{\prime \prime}\right|}\right)_{, i j}\left(\frac{1}{\left|\underline{x}^{\prime \prime}-\underline{x}^{\prime}\right|}\right)_{, k l} d V^{\prime \prime}=-2 \pi\left|\underline{x}-\underline{x}^{\prime}\right|_{, i j k l}
$$

Eq. 21 together with Eq. 13 (or Eq. 17) represent the two exact integral equations of the present time-incremental internal variables approach to be solved simultaneously in a given time step, using the properties of Green's tensor for isotropic properties of the homogeneous reference medium (i.e. the matrix phase in section 3). In the following, the Eshelby inclusion problem is first solved in the context of arbitrary anisotropic ellipsoidal Maxwellian inclusion embedded in an infinite isotropic Maxwellian matrix. Then, a homogenization Mori-Tanaka scheme for two-phase linear viscoelastic composites is developed. 


\section{Homogenization Mori-Tanaka scheme for two-phase linear viscoelastic composites}

\subsection{Linear viscoelastic Eshelby problem}

First, the strain concentration equations for the linear viscoelastic Eshelby problem are derived. The volume $V$ is constituted of an ellipsoidal inhomoheneity $I$ with volume $V_{I}$ embedded in an infinite matrix $M$ (here considered as the homogeneous reference medium 0 introduced in section 2) subjected to $\underset{\sim}{\dot{\varepsilon}^{M}}$ at remote boundaries. By denoting $\underset{\sim}{\dot{\varepsilon}^{I}}$ the spatial average of $\underset{\sim}{\dot{\varepsilon}}$ over the ellipsoidal inclusion $V_{I}, \underset{\sim}{\dot{\varepsilon}^{I}}$ is obtained from Eq. 13:

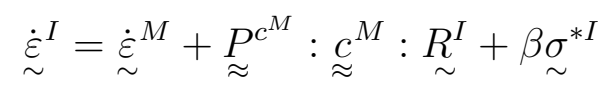

where constant $\beta$ is given by:

$$
\beta=\frac{3}{3 k^{M}+4 \mu^{M}}\left(\frac{k^{M}}{k_{v}^{M}}-\frac{\mu^{M}}{\mu_{v}^{M}}\right)
$$

and, $\underset{\sim}{\sigma^{I}}$, resp. $\underset{\sim}{\sigma^{* I}}$, is the spatial average of $\underset{\sim}{\sigma}$, resp. $\underset{\sim}{\sigma^{*}}$ over $V_{I}$, and:

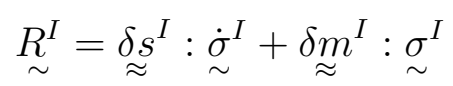

and, for any $\underline{x} \in V_{I}$, the fourth order tensor $\underset{\approx}{P^{c^{M}}}$ is defined as:

$$
\underset{\approx}{P^{c^{M}}}=\int_{V_{I}}{\underset{\approx}{\Gamma^{M}}}^{c^{\prime}}\left(\underline{x}-\underline{x}^{\prime}\right) d V^{\prime}
$$

$\underset{\approx}{P^{c^{M}}}$ is a uniform tensor due to the Eshelby's property for ellipsoidal inhomogeneities

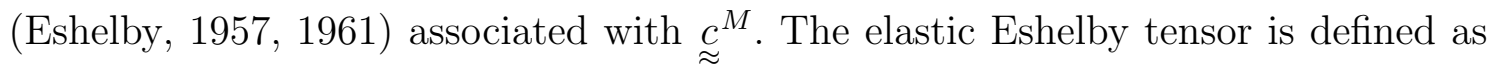

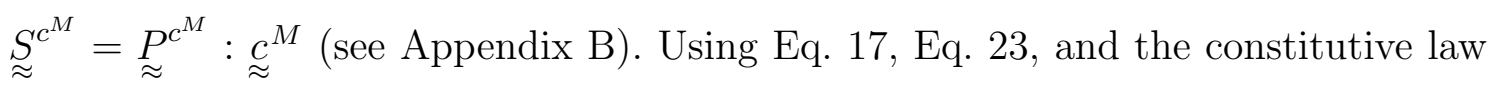
for the matrix phase yields:

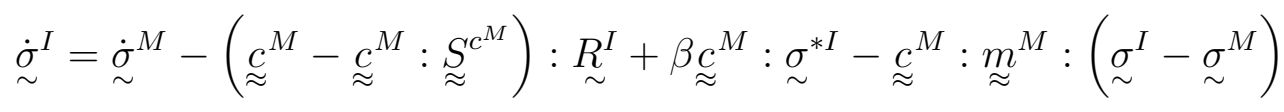


where $\underset{\sim}{\sigma^{M}}$ is the remote stress in the infinite matrix.

Using Eq. 21, $\underset{\sim}{\sigma^{* I}}$ is the solution of the following time differential equation:

$$
\dot{\sigma}_{i j}^{* I}=-\frac{\alpha^{c^{M}}}{4 \pi} \psi_{, i j k l}^{I} R_{k l}^{I}+\frac{3 \alpha^{c^{M}}}{2 \pi} \phi_{, i j}^{I} R_{m}^{I}-\frac{\alpha^{c^{M}}}{\alpha^{b^{M}}} \sigma_{i j}^{* I}
$$

In Eq. 28, $\phi^{I}$ and $\psi^{I}$ are harmonic and bi-harmonic potentials (Eshelby, 1957, 1961) related to the ellipsoidal inhomogeneity (see Appendix B). Introducing Eq. 23 in Eq. 28 yields (Berbenni et al., 2015):

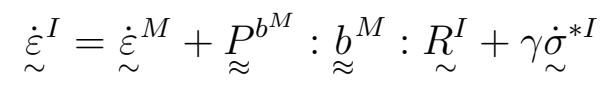

where constant $\gamma$ is given by:

$$
\gamma=\frac{3}{3 k_{v}^{M}+4 \mu_{v}{ }^{M}}\left(\frac{k_{v}{ }^{M}}{k^{M}}-\frac{\mu_{v}^{M}}{\mu^{M}}\right)
$$

and, where $\underset{\approx}{P^{b^{M}}}$ is a uniform tensor associated with $\underset{\approx}{b^{M}}$ :

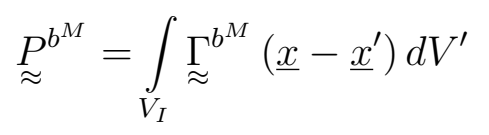

The anelastic Eshelby tensor is defined as $\underset{\approx}{S^{b^{M}}}=\underset{\approx}{P^{b^{M}}}: \underset{\approx}{b^{M}}$ (see Appendix B). Using Eqs. 2 together with Eq. 29 yields:

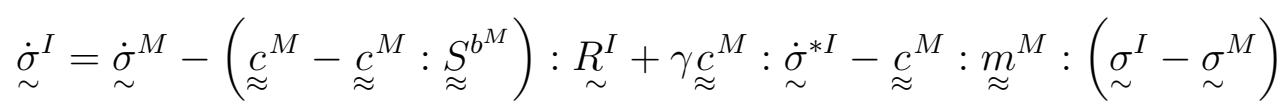

In the case of spherical inhomogeneities for applications to particulate composites in section 5, the expressions of $\underset{\approx}{P^{M}}$ and $\underset{\sim}{\dot{\sigma}^{* I}}$ are given in Appendix B.

\subsection{Homogenization Mori-Tanaka (MT) scheme for two-phase composites}

In the case of two-phase composite materials, the Representative Volume Element (RVE) is constituted of inclusions (phase $I$ ) with volume fraction $f$ embedded in 
a matrix phase $M$ with volume fraction $(1-f)$. The Mori-Tanaka (MT) homogenization scheme (Mori and Tanaka, 1973; Weng, 1984; Benveniste, 1987) is based on choosing the infinite homogeneous reference medium as the matrix phase $M$. This scheme is well adapted to estimate the effective properties of two-phase composites with an isotropic distribution of phases, provided the volume fraction $f$ of inclusions is not too large. The RVE is subjected at its boundary $\partial V$ to the homogeneous macroscopic strain rate here denoted $\underset{\sim}{\dot{E}}$ satisfying:

$$
\underset{\sim}{\dot{E}}=f \underset{\sim}{\dot{\varepsilon}^{I}}+(1-f) \underset{\sim}{\dot{\varepsilon}^{M}}
$$

The strain rate in the phase $I$ is obtained from Eq. 23 together with Eqs. 1 and Eq. 25:

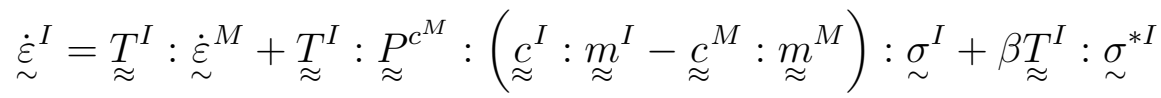

where the elastic tensor $\underset{\approx}{T}{ }^{I}$ (Benveniste, 1987) is given by:

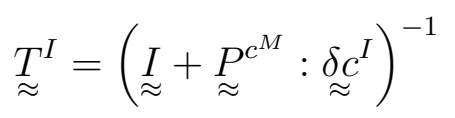

with $\underset{\approx}{\delta c^{I}}=\underset{\approx}{c^{I}}-\underset{\approx}{c^{M}}$

Using Eq. 34 together with Eq. 33 gives the strain rate in the matrix phase $M$ :

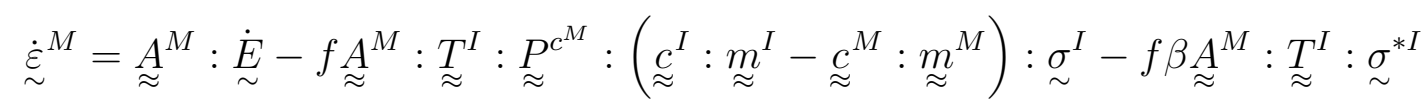

where $\underset{\approx}{A}$ is the elastic strain concentration tensor of the phase $M$ used to derive MT estimate in pure elasticity (Benveniste, 1987):

$$
\underset{\approx}{A^{M}}=\left[(1-f) \underset{\approx}{I}+f \underset{\approx}{T^{I}}\right]^{-1}
$$

The (average) stress rates in each phase $\underset{\sim}{\dot{\sigma}^{I}}$ and $\underset{\sim}{\dot{\sigma}^{M}}$ are obtained from Eqs. 34, 36 , and from the Hooke's law. Lastly, the overall stress rate $\underset{\sim}{\dot{\Sigma}}$ of the composite is 
incrementally solved from the stress rate averaging rule:

$$
\underset{\sim}{\dot{\Sigma}}=f \underset{\sim}{\dot{\sigma}^{I}}+(1-f) \underset{\sim}{\dot{\sigma}^{M}}
$$

Therefore, the effective behavior of the two-phase linear viscoelastic composite can be obtained from Eq. 38 together with Eq. 33. Considering the Laplace-Carson transform method for non ageing linear viscoelastic materials, the agreement of the present MT formulation (i.e. Hashin-Shtrikman estimates) with the MT estimate obtained from the correspondence principle (Wang and Weng, 1992) is proved in Appendix $\mathrm{C}$ for both strain and stress-based formulations.

\subsection{Discussion: comparison with other recent approaches}

Compared to the recent internal variable formulation proposed by Ricaud and Masson (2009) (case where the elastic properties of both phases are identical) and Masson et al. (2020), the present formulation also makes use of a fictitious linear (viscoelastic) comparison composite (LCC) and same modified secant formulation will be used, see section 4 . However, a major difference with their formulation is that, here there is no need to use the correspondence principle (Mandel, 1966) as in the equations (6-11) in Masson et al. (2020)) to find the time-incremental effective behavior of twophase linear viscoelastic composites. All the equations in this section were directly solved in the real time domain using the Eshelby tensors reported in Appendix B (isotropic matrix, general ellipsoidal inhomogeneity). It was only checked afterwards (see Appendix C) that the present equations agree with the MT estimates obtained from the correspondence principle for two-phase non-ageing viscoelastic composites. In contrast, Masson et al. (2020) started from Laplace-Carson (LC) transform of the stress concentration equation related to the MT estimate (see their equation (13)) to derive the time evolutions of averaged phase stresses (see their equation (15)). 
Finally, Masson et al. (2020) applied their method to an isotropic effective behavior with both isotropic phases (see their section 3), where LC inversions are straightforward to perform. In the present formulation, first, no inversion of LC transforms are needed to find the time evolutions of the overall and average phase responses, and, second, only the matrix phase $M$ is supposed to have isotropic properties, see Eqs. 7 and 8. Furthermore, the ellipsoidal non-spherical shape of the inhomogeneities can be considered by the present method, so that more general two-phase composites than particulate composites can be handled with the same set of equations as reported in this section. The method proposed by Masson et al. (2020) was explicitly formulated and used for the case of spherical inhomogeneities and isotropic effective behavior (see their equation (45) and their Appendix A). In comparison with the recent proposal of Cotelo et al. (2020), the present MT estimate is not based on a differential algebraic system of equations (DAS) using as primary variables the variances of the field fluctuations, but it makes use of a fictitious linear comparison viscoelastic composite for which the effective behavior is directly obtained in the time domain through Eqs. 33 to Eq. 38. In Cotelo et al. (2020), the time discretization scheme to approximate the stress rate is based on an implicit Euler scheme, so that an incremental stress potential is exploited using the incremental viscous compliance and strain-rate polarization tensors of each phase of the composite. Their differential equations can be applied to more general anisotropic behaviors than only isotropic ones. However, the applications in Cotelo et al. (2020) are shown for isotropic phases and isotropic microstructures using MT estimates which are easier to derive. In the present method, the case of two-phase elasto-viscoplastic (non linear viscoelastic) isotropic composites with elastic particles is considered with the present linear viscoelastic MT estimates, making use of a modified secant formulation, where the "effective stress" in the matrix phase $M$ is based on the second order moment of the deviatoric stress field. This methodology is explained in the next section. 


\section{A modified secant formulation for two-phase non linear visco-elastic isotropic composites with elastic particles}

To study the effect of non linear (possibly ageing) behavior on the overall composite responses, the MT scheme derived in section 3 is extended to non linear viscoelasticity, i.e. elasto-viscoplastic composites, using the linearized relationship described in Eq. 2. Here, one assumes that the matrix phase $M$ is isotropic elasto-viscoplastic and the particles $I$ are isotropic elastic. Therefore, $\underset{\approx}{m^{I}}=0$. The matrix phase $M$ exhibits a non linear incompressible viscoplastic behavior given by the following dissipation potential $\Phi^{v p}$ :

$$
{\underset{\sim}{\varepsilon^{v}}}^{v p^{M}}=\frac{\partial \Phi^{v p^{M}}}{\partial \sigma_{\sim}^{M}}
$$

where $\sigma_{\sim}^{\prime}$ is the deviatoric stress tensor, and $\Phi^{v p}$ is given for $M$ by a the power law:

$$
\Phi^{v p^{M}}\left(\underset{\sim}{\sigma^{M}}\right)=\frac{\dot{\varepsilon_{0}} \sigma_{0}}{n+1}\left(\frac{<\sigma_{e q}^{M}-\sigma_{Y}>}{\sigma_{0}}\right)^{n+1}
$$

where $n$ is an exponent such that $m=1 / n$ is the strain-rate sensitivity of the matrix phase, $\sigma_{e q}^{M}$ is the equivalent Von Mises stress (or effective stress), $\dot{\varepsilon_{0}}$ is a reference strain rate, $\sigma_{0}, \sigma_{Y}$ (threshold stress) are material's parameters. $\langle x\rangle$ denotes the positive part of $x$, i.e. $\langle x\rangle=\max (x, 0)$. For $m \neq 1$, the behavior is non linear, which is the case for metallic alloys for example. The elastic compliance tensor $\underset{\approx}{s}$ is isotropic for both phases $I$ and $M$, which are elastically compressible:

$$
\underset{\approx}{\stackrel{I}{\approx}, M}=\frac{1}{3 k^{I, M}} \underset{\approx}{J}+\frac{1}{2 \mu^{I, M}} \underset{\approx}{K}
$$

where $k^{I, M}$ ad $\mu^{I, M}$ are the elastic bulk and shear moduli for phases $I$ and $M$, respectively. For the matrix phase $M$, Eq. 39 also writes:

$$
\dot{\sim}^{M}=\underset{\approx}{s}{ }^{M}: \dot{\sim}^{M}+\frac{1}{2 \mu_{v} M} \sigma_{\sim}{ }^{M}
$$


where $\mu_{v}{ }^{M}$ is the non-linear shear viscoplastic modulus:

$$
\mu_{v}{ }^{M}=\frac{\sigma_{e q}^{M}}{3 \dot{\varepsilon_{0}}}\left(\frac{\sigma_{0}}{<\sigma_{e q}^{M}-\sigma_{Y}>}\right)^{n}
$$

The "classical secant" (denoted "SEC") linearization procedure only considers the first order stress moments (i.e. the phase average stresses) and tends to overestimate the effective behavior. In the case of the SEC linearization, the linearized viscoplastic compliance in the matrix phase $M$ becomes:

$$
\underset{\approx}{m}{ }^{M}=\frac{1}{2 \mu_{v}} \underset{\approx}{K}
$$

where $\underset{\approx}{m}{ }^{M}$ is the classic secant compliance tensor. For this classic formulation, $\underset{\approx}{m^{M}}$ depends on the first order moment of deviatoric stress field with an effective stress given by ${\overline{\sigma_{e q}}}^{M}$ defined as:

$$
\sigma_{e q}^{M}={\overline{\sigma_{e q}}}^{M}=\left(\frac{3}{2} \underset{\sim}{\bar{\sigma}^{\prime}}{ }^{M}:{\overline{\sigma^{\prime}}}^{M}\right)^{1 / 2}
$$

In contrast, the so-called "modified secant" formulation (denoted "SEC MODIF") includes second order stress moments and leads to better estimates for voided materials and particulate composites with rigid inclusions (Qiu and Weng, 1991; Suquet, 1995; Hu, 1996; Ponte Castañeda and Suquet, 1998). The "SEC MODIF" linearization procedure was developed to better account for intra-phase heterogeneities in non linear composites and makes use of an isotropic LCC. For the computation of second moments of stress tensor for isotropic linear viscoelastic composites using MT estimate, only the trace $(i j i j)$ of the second-order moment of stress field is needed (Masson et al., 2020). As the MT estimate is used in the present formulation, the stress field in the inclusion phase $I$ only needs first moments of stress field. Therefore, the present "SEC MODIF" formulation is based on the estimate of the "effective stress" in the matrix phase $\sigma_{e q}^{M}$ defined as the square-root of the (ijij) trace of the 
deviatoric part of the second stress moment:

$$
\sigma_{e q}^{M}={\overline{\overline{\sigma_{e q}}}}^{M}=\left(\frac{3}{2} \overline{\bar{S}}^{M}\right)^{1 / 2}
$$

where $\overline{\bar{S}}^{M}=\bar{\sim}_{\sim}^{\sigma_{\sim}^{\prime}}{ }^{M}$.

In order to evaluate the time evolution of ${\overline{\overline{\sigma_{e q}}}}^{M}$, a direct method is used as the one shown in Masson et al. (2020) (for compressible two-phase composites), Li and Weng (1997) (for compressible two-phase composites but specified to creep loadings ) and Badulescu et al. (2015) (for incompressible two-phase composites). The method is based on the application of the Hill's lemma (Hill, 1967):

$$
\underset{\sim}{\sigma: \underset{\sim}{\dot{\varepsilon}}}=\bar{\sim}: \bar{\sim}
$$

Neglecting the second order moment of the hydrostatic stress field in the matrix phase $M$ like in Li and Weng (1997) and Masson et al. (2020), straightforward algebraic manipulations lead to the following time evolution equation for $\overline{\bar{S}}^{M}$ :

$$
\dot{\overline{\bar{S}}}^{M}+\frac{2}{\tau^{M}} \overline{\bar{S}}^{M}=4 \mu^{M} \frac{X}{1-f}
$$

where $\tau^{M}=\frac{\mu_{v} M}{\mu^{M}}$ is the relaxation time of the matrix phase, and $X$ is given by:

$$
X=\underset{\sim}{\bar{\sigma}}: \underset{\sim}{\bar{\varepsilon}}-\frac{f}{2 \mu^{I}} \underset{\sim}{\dot{\sigma^{\prime}}}{ }^{I}: \bar{\sim}_{\sim}^{I}-\sum_{r} \frac{f^{(r)}}{k^{(r)}}{\overline{\sigma_{m}}}^{(r)}{\overline{\sigma_{m}}}^{(r)}
$$

with $(r)=I, M, f^{I}=f, f^{M}=1-f$. At t $=0$, it is assumed that $\overline{\bar{S}}^{M}=0$. Then, the time evolution of $\overline{\bar{S}}^{M}$ can be calculated using Eq. 48 together with Eq. 49 to derive the "SEC MODIF" compliance tensor of the matrix phase $\underset{\approx}{m}\left({\overline{\overline{\sigma_{e q}}}}^{M}\right)$ (Eq. 44) from the "effective secant viscosity" $\mu_{v}\left({\overline{\overline{\sigma_{e q}}}}^{M}\right)$ (Eq. 43) using Eq. 46.

In the following section, the present time-incremental MT homogenization scheme for elasto-viscoplastic particulate composites based on the equations derived in sections 3 and 4 will be called "MT-SEC-MODIF" formulation. In the next section, numerical 
results are reported and discussed. All numerical calculations for the present "MTSEC-MODIF" formulation presented in this work have been performed with a $\mathrm{C}++$ code in the Simula + environment (www.lem3.fr/collard/pub/simula+/doc/html).

\section{Results for two-phase particulate composite materials with a non lin- ear viscoelastic matrix phase reinforced by spherical elastic particles}

\subsection{Uniaxial tensile loading and strain rate sensitivity effects}

In this first application, the two-phase composite contains a linear elastic inclusion phase $I$ and an elasto-viscoplastic matrix phase $M$ with a power-law dissipative potential in the form of Eq. 40 with zero threshold stress $\left(\sigma_{Y}=0\right)$. The materials parameters for both phases are given in Table 1. The volume fraction of the inclusion phase is set to $f=0.25$. One considers a uniaxial tensile loading in the direction parallel to $\underline{e}_{3}$ where the applied stress tensor is given by:

$$
\underset{\sim}{\Sigma}(t)=\Sigma_{33}(t) \underline{e}_{3} \otimes \underline{e}_{3}
$$

The applied strain rate is fixed to $\dot{E}_{33}=10^{-2} s^{-1}$. In this simulation, at each time step, the non zero overall stress rate component $\dot{\Sigma}_{33}$ is computed as well as the overall strain rate tensor $\underset{\sim}{\dot{E}}$, using the effective behavior relationships (Eqs. 33 and $38)$.

This case was first investigated by Lahellec and Suquet (2007b) using a "modified secant" formulation in an "Effective Internal Variable" (EIV) approach, where FEM calculations were performed to assess the homogenization model. The FEM calculations were based on a periodic unit cell approximated by a cylindrical unit cell containing an inclusion. Later, Czarnota et al. (2015) reproduced the same results, but also considers larger RVEs with 30 inclusions (instead of one inclusion). 
Therefore, FEM calculations with 30 inclusions are considered as reference full-field calculations for this application. In addition, in the linear case $n=1$, the LC analytical solution for the MT homogenization was also reported by Czarnota et al. (2015).

First of all, the numerical results for the effective behavior and the phase responses given by the present "MT-SEC-MODIF" formulation are reported in Figure 1 for a linear viscoelastic matrix $((\mathrm{a}) n=1)$ and for a non linear elasto-viscoplastic matrix ((b) $n=10)$. Compared to $n=1$ (larger strain rate sensitivity), the transition between elastic and viscoplastic regimes is very sharp in the case of $n=10$ (lower strain rate sensitivity) for both macroscopic and matrix behaviors. In Figure 2, some comparisons are made with the Mori-Tanaka (MT) estimate using exact inverse Laplace-Carson (LC) formula for $n=1$ and FEM calculations with 30 inclusions in the RVE from Czarnota et al. (2015) for $n=1,10,100$. A very good match is obtained in the elastic regime. For the linear case $n=1$, the present MT model underestimates the overall response with respect to full-field FEM calculations at large times, i.e. in the viscoplastic regime, which was already observed by Lahellec and Suquet (2007b). But, it exactly matches the theoretical result given by inverse LC transform for the MT homogenization scheme for all times. The fact that the present formulation exactly matches the inverse LC response was already theoretically proved in Berbenni et al. (2015) and in Appendix C. The estimates of the effective behavior given by the "MT-SEC-MODIF" formulation for non linear cases ( $n=10$ and $n=100)$ are very close to the FEM calculation results. 


\begin{tabular}{cccccccc}
\hline Phase & $E(\mathrm{GPa})$ & $\nu$ & $\dot{\varepsilon_{0}}\left(s^{-1}\right)$ & $\sigma_{0}(\mathrm{MPa})$ & $\sigma_{Y}(\mathrm{MPa})$ & $n$ \\
\hline Matrix (elasto-viscoplastic) & 70 & 0.3 & $10^{-2}$ & 480 & 0 & $1,10,100$ \\
\hline Inclusion (elastic) & 400 & 0.2 & $/$ & $/$ & $/$ & $/$ \\
\hline Table 1 & & & & &
\end{tabular}

Table 1

Materials parameters for two-phase particulate composites with a non linear elastoviscoplastic matrix phase reinforced by spherical elastic particles $(f=0.25)$. For these simulations, different strain rate sensitivities are considered: $m=1,0.1,0.01$. For isotropic elastic parameters, $E$ is the elastic Young's modulus and $\nu$ is the elastic Poisson ratio.

(a) $n=1$

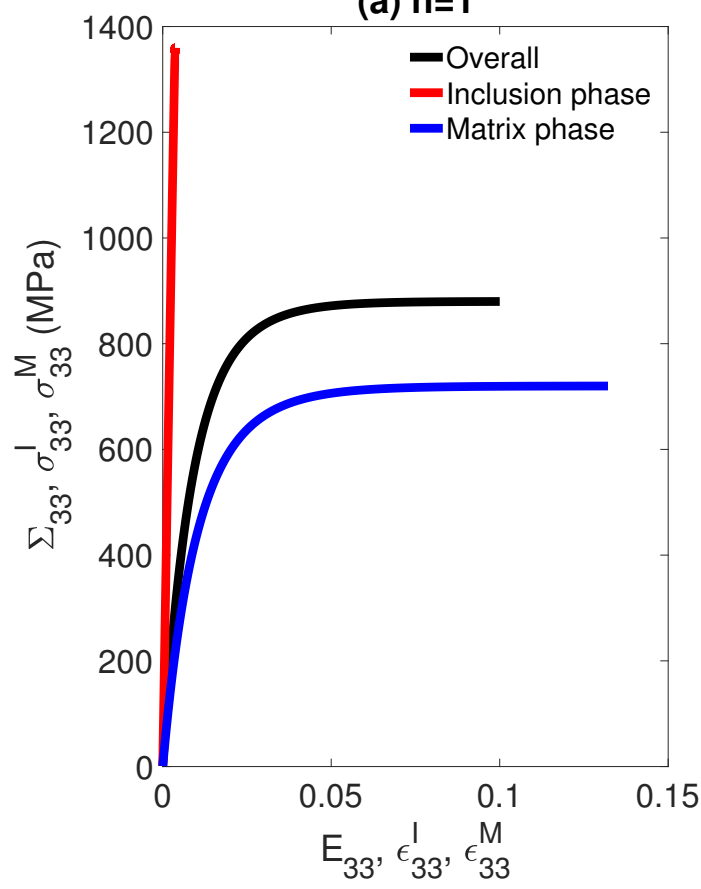

(b) $\mathrm{n}=\mathbf{1 0}$

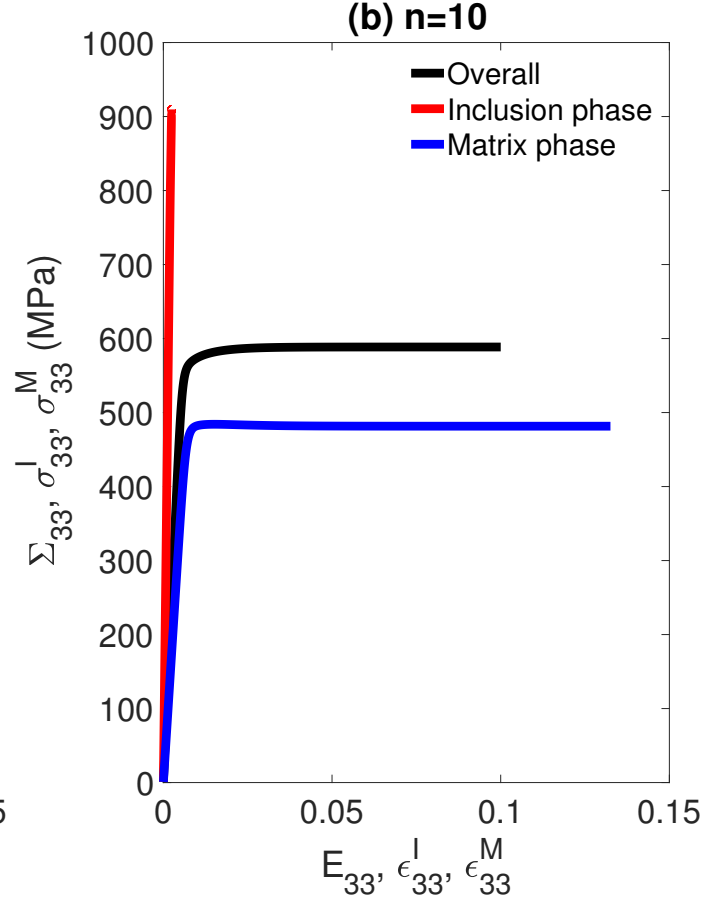

Figure 1. Numerical results given by the "MT-SEC-MODIF" formulation: overall (effective behavior, black solid line), inclusion phase (red solid line), matrix phase (blue solid line) monotonic stress-strain responses of a two-phase composite with spherical elastic inclusions of volume fraction $f=0.25$ and with a linear viscoelastic matrix (a) $n=1$, and a non-linear elasto-viscoplastic matrix (b) $n=10$, see Table 1 for materials parameters.

\subsection{Strain rate effects, non-monotonic and non-radial loadings}

In the following applications, the two-phase composite is constituted of a linear elastic inclusion phase $I$ and an elasto- 28 coplastic matrix phase $M$ with a power- 


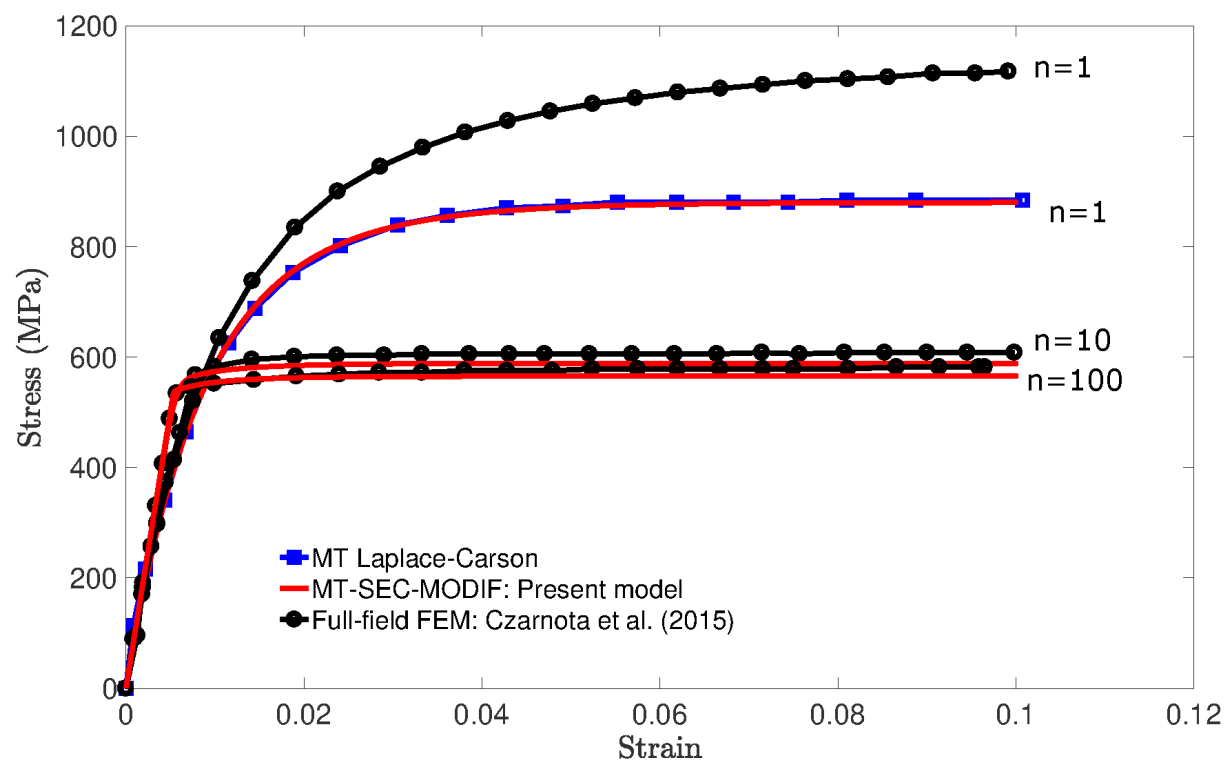

Figure 2. Numerical results given by the "MT-SEC-MODIF" formulation: predicted overall stress $\left(\Sigma_{33}\right) /$ strain $\left(E_{33}\right)$ responses of a two-phase composite with spherical elastic inclusions of volume fraction $f=0.25$ and an elasto-viscoplastic matrix with three power exponents: $n=1,10,100$ (i.e. three strain sensitivities), see Table 1 for materials parameters of both phases. Comparisons are made with the Mori-Tanaka (MT) estimate using the exact inverse Laplace-Carson (LC) formula for $n=1$ and with FEM calculations with 30 inclusions in the RVE from Czarnota et al. (2015) for $n=1,10,100$.

law dissipative potential in the form of Eq. 40 with threshold stress $\sigma_{Y}=100 M P a$. The materials parameters for both phases are given in Table 2. The volume fraction of the inclusion phase is set to $f=0.17$.

A monotonic loading with applied strain tensor at different strain rate magnitudes is considered:

$$
\underset{\sim}{E}(t)=E_{33}(t)\left(-0.5 \underline{e}_{1} \otimes \underline{e}_{1}-0.5 \underline{e}_{2} \otimes \underline{e}_{2}+\underline{e}_{3} \otimes \underline{e}_{3}\right)
$$

where the applied strain rates $\dot{E}_{33}$ are $0.012 s^{-1}, 0.12 s^{-1}, 1.2 s^{-1}, 12 s^{-1}, 120 s^{-1}$ and $1200 s^{-1}$, respectively. In Figure 3, a very good agreement with the full-field FFT calculations performed by Lahellec and Suquet (2013) is shown. Thus, the overall 


\begin{tabular}{cccccccc}
\hline Phase & $k(\mathrm{GPa})$ & $\mu(\mathrm{GPa})$ & $\dot{\varepsilon_{0}}\left(s^{-1}\right)$ & $\sigma_{0}(\mathrm{MPa})$ & $\sigma_{Y}(\mathrm{MPa})$ & $n$ \\
\hline Matrix (elasto-viscoplastic) & 10 & 3 & 1 & 100 & 100 & $10 / 3$ \\
\hline Inclusion (elastic) & 20 & 6 & $/$ & $/$ & $/$ & $/$ \\
\hline Table 2 & & & & &
\end{tabular}

Materials parameters for a two-phase particulate composite with a non linear elastoviscoplastic matrix phase reinforced by spherical elastic particles $(f=0.17)$. The strain rate sensitivity of the matrix is $m=0.3$. For isotropic elastic parameters, $k$ is the elastic bulk modulus and $\mu$ is the elastic shear modulus.

strain rate effect on the monotonic response of the particulate composite is well predicted by the present "MT-SEC-MODIF" formulation.

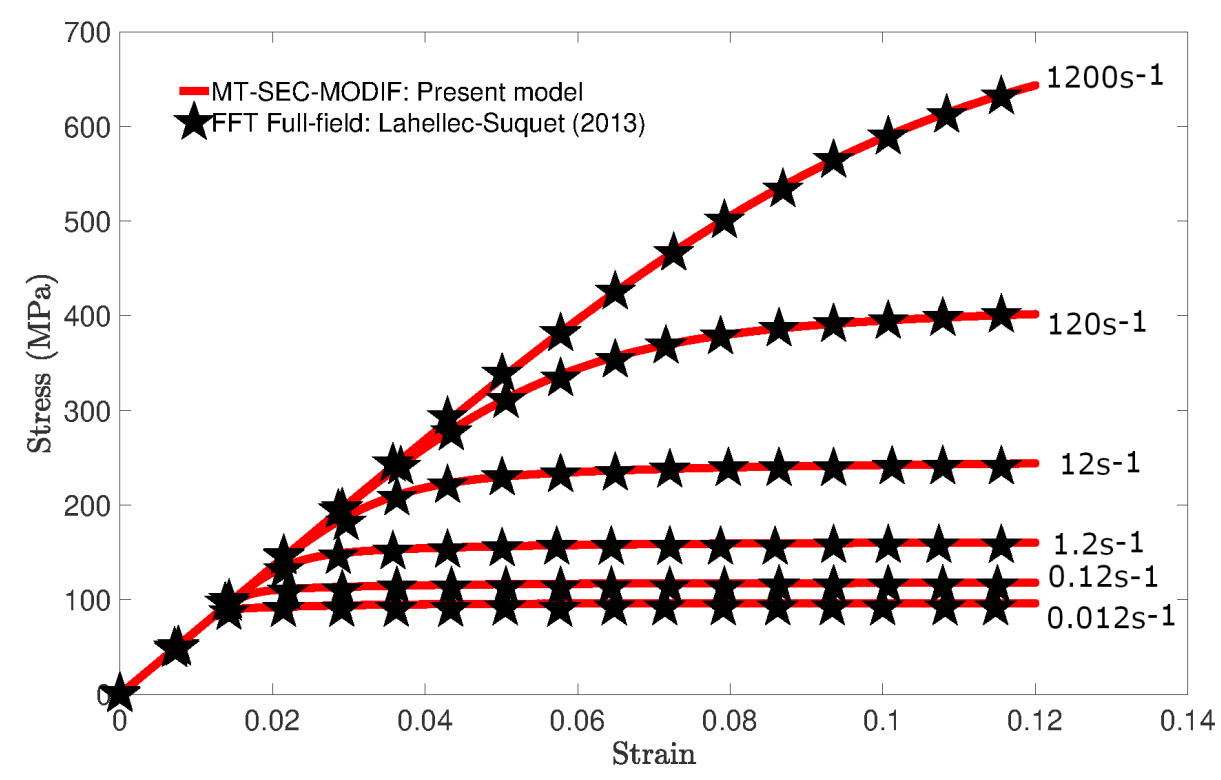

Figure 3. Numerical results given by the "MT-SEC-MODIF" formulation: monotonic overall stress $\left(\Sigma_{33}\right) /$ strain $\left(E_{33}\right)$ responses obtained at different applied strain rates $\dot{E}_{33}=0.012 s^{-1}, 0.12 s^{-1}, 1.2 s^{-1}, 12 s^{-1}, 120 s^{-1}, 1200 s^{-1}$ (red solid lines) . Comparisons with the full-field FFT calculations reported in Lahellec and Suquet (2013) (star symbols).

Now, the same strain-controlled radial loading as Eq. 51 is used, but for non- 
monotonic (cyclic) loading. The applied radial strain rate history with two changes in the sign of $\dot{E}_{33}$ is the following:

$$
\begin{aligned}
& \dot{E}_{33}(t)=12 \cdot 10^{-3} s^{-1} \text { for } 0 s \leq t \leq 10 s \\
& \dot{E}_{33}(t)=-12 \cdot 10^{-3} s^{-1} \text { for } 10 s \leq t \leq 30 s \\
& \dot{E}_{33}(t)=12 \cdot 10^{-3} s^{-1} \text { for } 30 s \leq t \leq 40 s
\end{aligned}
$$

Figure 4 represents the overall cyclic behavior obtained with the "MT-SEC-MODIF" formulation (red solid lines). These responses are compared to the full-field results of Lahellec and Suquet (2013) (star symbols) and the Maxwell approximation (blue solid line), which consists in the homogenization of elastic and plastic parts separately to end with an effective constitutive law of Maxwell type for the composite. It is seen that the agreement of the present model is rather good for the overall behavior. In contrast, it is noted that the Maxwell approximation predicts an overall stress higher than the viscoplastic limit, right after the elasto-viscoplastic transition, which confirms that the effective (overall) behavior of a composite of Maxwellian constituents is no more Maxwellian. Figure 5 represents the averaged phase responses obtained with the "MT-SEC-MODIF" formulation (red solid lines) compared with the full-field results of Lahellec and Suquet (2013) (star symbols). A very good agreement with the full-field reference results is obtained during the whole cycle for the average stress in the matrix phase $M$. For the inclusion phase $I$, the stress is a little bit overestimated by the present mean field model. In order to explain the possible origin of these differences, Figure 6 reports the time-evolution of the stress fluctuations over the matrix phase $M$ with the "MT-SEC-MODIF" formulation (red solid lines), which is computed as $\sqrt{\overline{\bar{S}^{M}-\bar{\sigma}_{\sim} M}: \bar{\sim}_{\sim}^{\sigma^{M}}}$ following Lahellec and Suquet (2013) and Masson et al. (2020). It is then seen that the the level of stress fluctuations in $M$ is relatively high for this composite, and, the predictions of the present model are not so far from the full-field results of Lahellec and Suquet 
(2013) (star symbols). The deviation from the FFT reference results stems from the fact that the matrix stress fluctuations are certainly not correctly estimated due to the absence of matrix hydrostatic stress fluctuations in the model, see Eqs. 48 and 49. Furthermore, it is noted that during a short period of time between loading sequences, the matrix stress field fluctuations vanish, which is not observed with the full field results. For comparison, the same example with incompressible phases is considered by setting infinite bulk moduli in both phases (purely deviatoric behavior for both phases), so that the matrix hydrostatic stress fluctuations automatically vanish. In this incompressible case shown in Figure 6 (blue dashed lines), the matrix stress field fluctuations do not vanish anymore in these short finite time intervals. However, it is highlighted that, apart from these short time intervals, the present "MT-SEC-MODIF" formulation gives better predictions than the incompressible case in the elastic-viscoplastic transition.

A last application concerns the application of the present model to a non-radial loading for the same materials parameters (see Table 2). Such non-radial loading paths were extensively studied essentially for elasto-plastic composites in Lahellec and Suquet (2013) and for elasto-viscoplastic composites in Masson et al. (2020). For this problem, full-field FFT results reported in Masson et al. (2020) serve as reference results to assess the present "MT-SEC-MODIF" formulation.

The strain-controlled non-radial loading history is given by:

$$
\begin{aligned}
& \underset{\sim}{E}(t)=E_{33}(t)\left(-0.5 \underline{e}_{1} \otimes \underline{e}_{1}-0.5 \underline{e}_{2} \otimes \underline{e}_{2}+\underline{e}_{3} \otimes \underline{e}_{3}\right) \\
& +E_{13}(t)\left(\underline{e}_{1} \otimes \underline{e}_{3}+\underline{e}_{3} \otimes \underline{e}_{1}+\underline{e}_{2} \otimes \underline{e}_{3}+\underline{e}_{3} \otimes \underline{e}_{2}\right)
\end{aligned}
$$




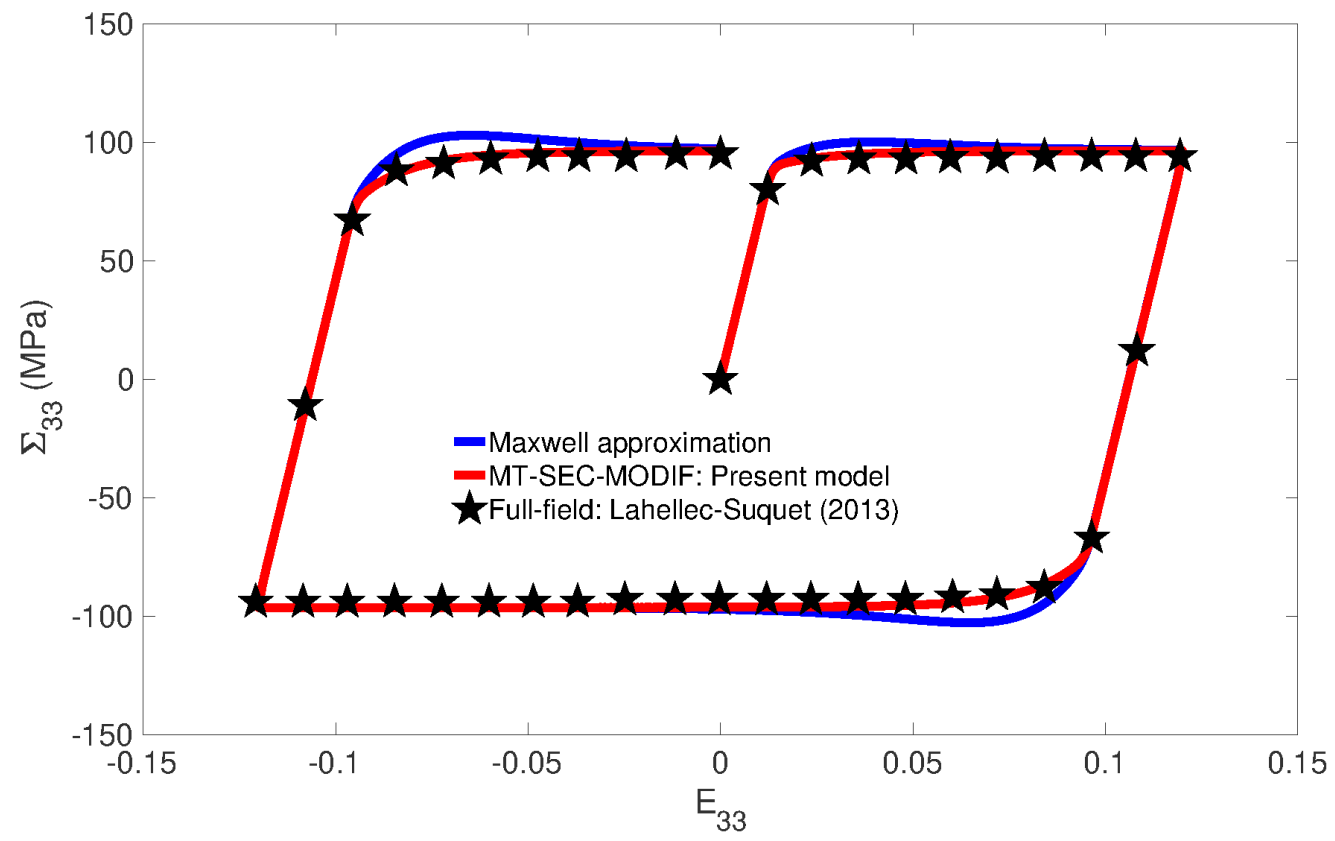

Figure 4. Numerical results given by the "MT-SEC-MODIF" formulation: overall stress-strain responses (red solid lines) obtained during a non-monotonic (cyclic) loading for a two-phase composite with spherical elastic inclusions of volume fraction $f=0.17$ and with a non-linear elasto-viscoplastic matrix $(n=10 / 3)$. Comparisons with the full-field FFT calculations reported in Lahellec and Suquet (2013) (star symbols) and the Maxwell approximation (blue solid lines).

where the time evolutions of prescribed strain rates $\dot{E}_{33}$ and $\dot{E}_{13}$ are:

$$
\begin{aligned}
& \dot{E}_{33}(t)=12 \cdot 10^{-3} s^{-1}, \dot{E}_{13}(t)=0 s^{-1} \text { for } 0 s \leq t \leq 10 s \\
& \dot{E}_{33}(t)=0 s^{-1}, \dot{E}_{13}(t)=7.5 \cdot 10^{-3} s^{-1} \text { for } 10 s \leq t \leq 20 s \\
& \dot{E}_{33}(t)=-12 \cdot 10^{-3} s^{-1}, \dot{E}_{13}(t)=0 s^{-1} \text { for } 20 s \leq t \leq 30 s \\
& \dot{E}_{33}(t)=0 s^{-1}, \dot{E}_{13}(t)=-7.5 \cdot 10^{-3} s^{-1} \text { for } 30 s \leq t \leq 40 s
\end{aligned}
$$

Because of the material's isotropy, this strain loading path involves a rotation of the principal strain axis and therefore a rotation of the principal stress axis in the plastic regime, as explained in Lahellec and Suquet (2013).

Figure 7 reports the predictions of the present "MT-SEC-MODIF" formulation regarding the overall shear stress $\Sigma_{13}$ as a function of the overall axial stress $\Sigma_{33}$ during 


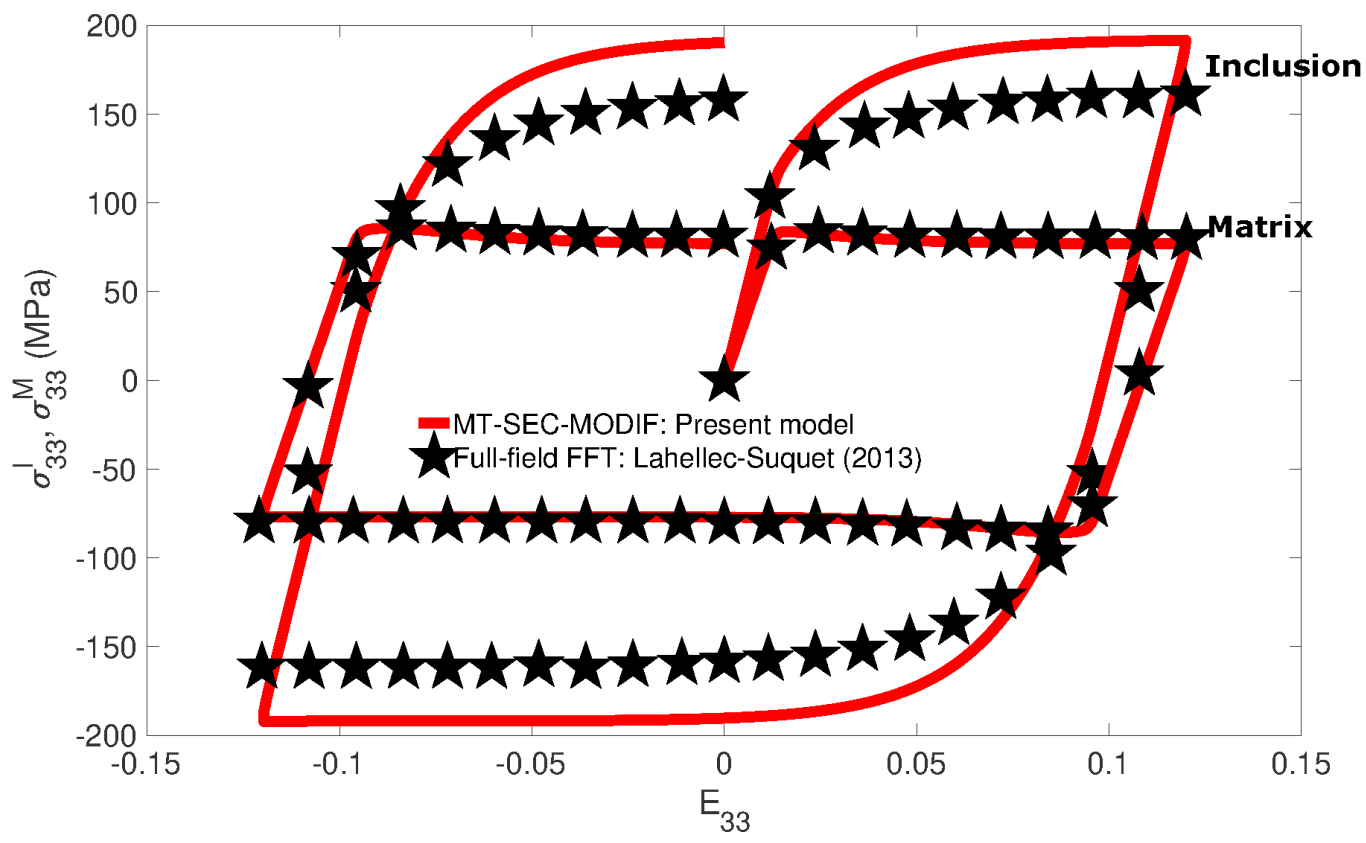

Figure 5. Numerical results given by the "MT-SEC-MODIF" formulation: averaged inclusion phase and matrix phase stress-strain responses (red solid lines) obtained during a non-monotonic (cyclic) loading for a two-phase composite with spherical elastic inclusions of volume fraction $f=0.17$ and with a non-linear elasto-viscoplastic matrix $(n=10 / 3)$. Comparisons with the full-field FFT calculations reported in Lahellec and Suquet (2013) (star symbols).

non-radial loadings given by Eqs. 53 and 54. In comparison with the full-field calculations (Masson et al., 2020), the model gives relatively good results, which are similar to the "modified secant" formulation developed by (Masson et al., 2020). 


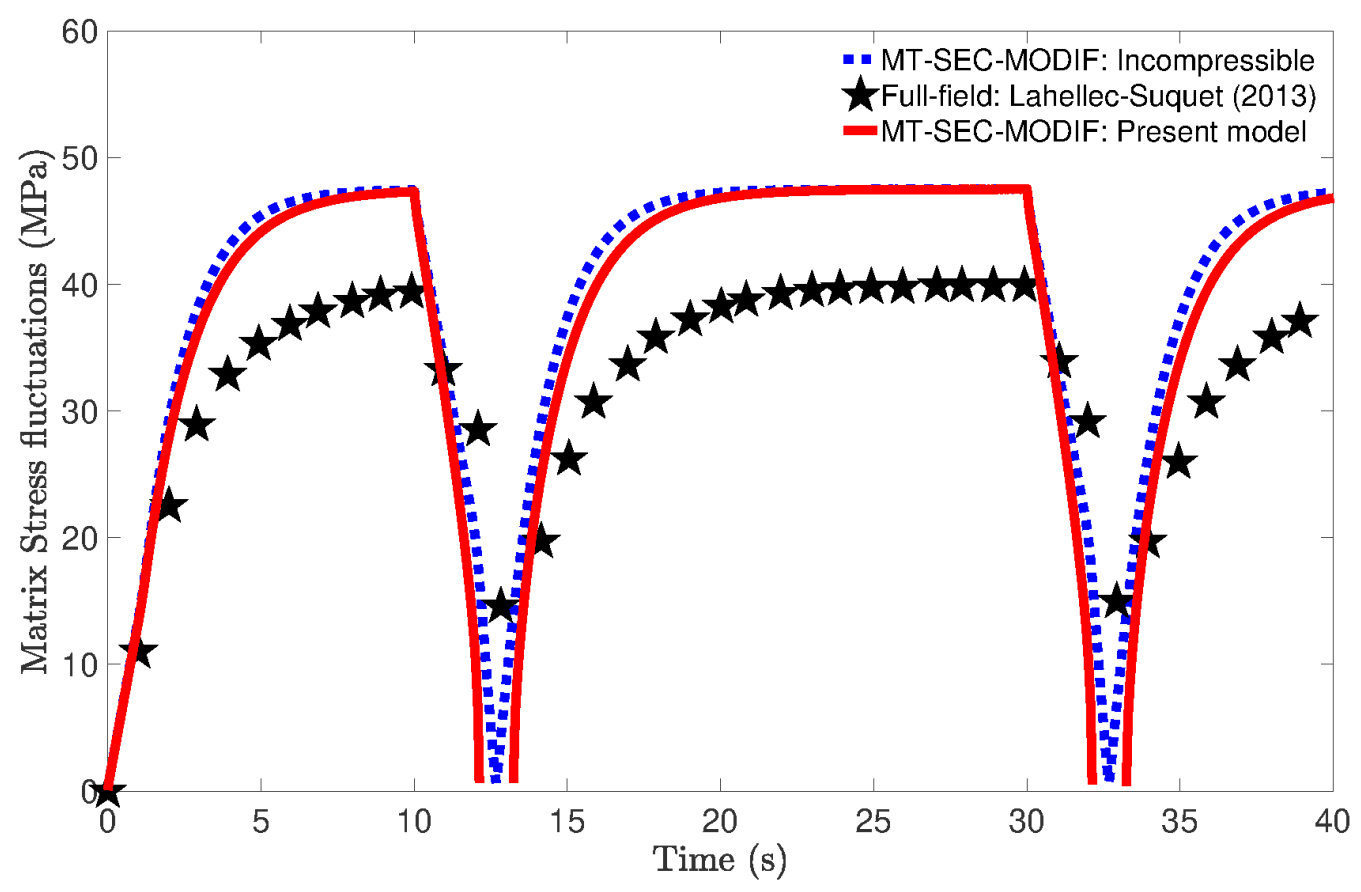

Figure 6. Numerical results given by the "MT-SEC-MODIF" formulation: time-evolution of the stress fluctuations over the matrix phase (red solid lines). Comparisons with the full-field FFT calculations reported in Lahellec and Suquet (2013) (star symbols) and the purely incompressible case (blue dashed lines).

\section{Conclusions and future studies}

A time-incremental internal variables homogenization scheme for non-linear viscoelastic (elasto-viscoplastic) isotropic two-phase particulate composite materials has been proposed. The formulation is based on a solution for the linear viscoelastic (Maxwellian) Eshelby problem assuming isotropic elastic and viscoplastic properties for the matrix phase. The homogenization Mori-Tanaka (MT) scheme is used for two-phase linear viscoelastic composites. The agreement of the present formulation with the MT scheme obtained from the correspondence principle (Wang and Weng, 1992), i.e. the Hashin-Shtrikman estimates in linear viscoelasticity, was proved for both strain and stress-based formulations. The extension to non-linear behavior is based on a "modified secant" formulation with an approximation to compute the second order moment of the deviatoric stress field in the matrix phase (the so-called 


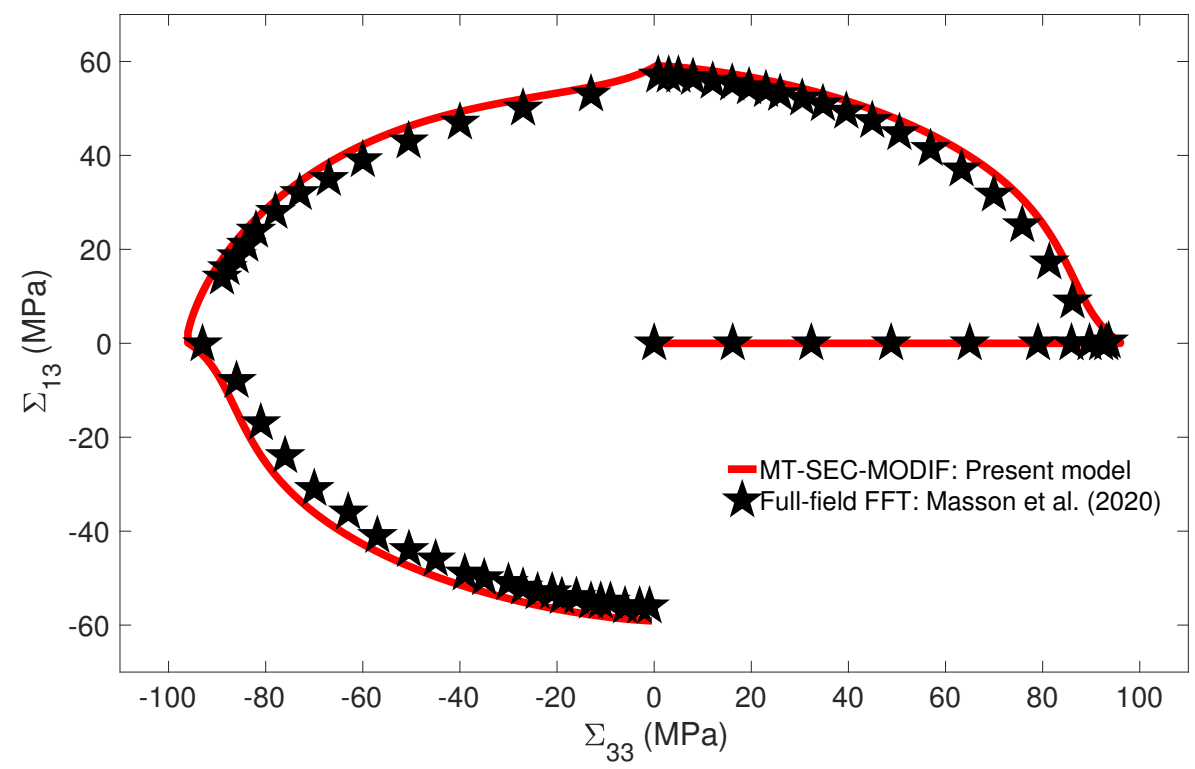

Figure 7. Numerical results given by the "MT-SEC-MODIF" formulation: evolution of the overall shear stress $\Sigma_{13}$ as a function of axial overall stress $\Sigma_{33}$ during non-radial loadings (red solid lines) given by Eqs. 53 and 54. Comparisons with the full-field FFT calculations reported in Masson et al. (2020) (star symbols).

"MT-SEC-MODIF" formulation in this work) in order to obtain the linear comparison composite properties.

In comparison with the approach of Masson et al. (2020) using the same "modified secant" formulation, the present model overcomes the use of the correspondence principle and Laplace-Carson inversions to formulate time evolutions of phases stresses and overall behavior. It gives quite accurate results regarding the effective behavior for such non-linear two-phase microstructures, which are similar to recent variational approaches methods for determining uniaxial tensile responses, strain rate sensitive responses and non-monotonic or non-radial responses.

In comparisons with the most recent differential variational methods (Cotelo et al., 2020; Das and Ponte Castañeda, 2021) based on the IVP of Agoras et al. (2016), the development and the applications of the present time-incremental homogeniza- 
tion scheme to other composites with more complex morphologies (fibers, plate like inclusions...) or anisotropic materials (polycrystals) is left for future studies. Indeed, the computation of the fluctuations of the stress field in the phases is a key issue to extend the proposed formulation to non linear viscoelastic polycrystals.

\section{Appendix}

\section{A Derivation of Eq. 22}

In this appendix, the objective is to prove Eq. 22 using the Fourier transform method. Let us denote $H_{i j k l}$ the following integral:

$$
H_{i j k l}=\int_{V}\left(\frac{1}{\left|\underline{x}-\underline{x}^{\prime \prime}\right|}\right)_{, i j}\left(\frac{1}{\left|\underline{x}^{\prime \prime}-\underline{x}^{\prime}\right|}\right)_{, k l} d V^{\prime \prime}
$$

Let $\underline{\xi}$ be the Fourier vector, then $\left(\frac{1}{\left|\underline{x}-\underline{x}^{\prime}\right|}\right)_{, i j}$ and $\left|\underline{x}-\underline{x}^{\prime}\right|_{, i j k l}$ are defined by:

$$
\begin{aligned}
\left(\frac{1}{\left|\underline{x}-\underline{x}^{\prime}\right|}\right)_{, i j} & =-\frac{1}{2 \pi^{2}} \int_{V_{\xi}} \frac{\xi_{i} \xi_{j}}{\xi^{2}} \exp \left(\underline{\mathbf{i}} \underline{\xi} \cdot\left(\underline{x}-\underline{x}^{\prime}\right)\right) d V_{\xi} \\
\left|\underline{x}-\underline{x}^{\prime}\right|_{, i j k l} & =-\frac{1}{\pi^{2}} \int_{V_{\xi}} \frac{\xi_{i} \xi_{j} \xi_{k} \xi_{l}}{\xi^{4}} \exp \left(\underline{\mathbf{i}} \cdot\left(\underline{x}-\underline{x}^{\prime}\right)\right) d V_{\xi}
\end{aligned}
$$

where $\mathbf{i}$ is the imaginary unit and $\xi$ is the magnitude of $\underline{\xi}$, i.e. $\sqrt{\xi_{i} \xi_{i}}$. From Eq. A.2,

$$
H_{i j k l}=\frac{1}{4 \pi^{4}} \int_{V} \int_{V_{\xi}^{\prime}} \frac{\xi_{i}^{\prime} \xi_{j}^{\prime}}{\xi^{\prime 2}} \exp \left(\underline{\mathbf{i}} \underline{\xi}^{\prime} \cdot\left(\underline{x}-\underline{x}^{\prime \prime}\right)\right) d V_{\xi^{\prime}} \int_{V_{\xi}} \frac{\xi_{k} \xi_{l}}{\xi^{2}} \exp \left(\underline{\mathbf{i}} \underline{\xi} \cdot\left(\underline{x}^{\prime \prime}-\underline{x}^{\prime}\right)\right) d V_{\xi} d V^{\prime \prime}
$$

Using the classic Fourier transform formula: $\int_{V} \exp \left(\mathbf{i}\left(\underline{\xi}-\underline{\xi^{\prime}}\right) \cdot \underline{x}^{\prime \prime}\right) d V^{\prime \prime}=8 \pi^{3} \delta\left(\underline{\xi}-\underline{\xi^{\prime}}\right)$, 
where $\delta\left(\underline{\xi}-\underline{\xi^{\prime}}\right)$ is the three-dimensional delta Dirac function, Eq. A.4 becomes:

$$
H_{i j k l}=\frac{2}{\pi} \int_{V_{\xi}} \int_{V_{\xi}^{\prime}} \delta\left(\underline{\xi}-\underline{\xi^{\prime}}\right) \frac{\xi_{i}^{\prime} \xi_{j}^{\prime}}{\xi^{\prime 2}} \frac{\xi_{k} \xi_{l}}{\xi^{2}} \exp \left(\underline{\mathbf{i}} \underline{\xi}^{\prime} \cdot \underline{x}\right) \exp \left(-\mathbf{i} \underline{\xi} \cdot \underline{x^{\prime}}\right) d V_{\xi^{\prime}} d V_{\xi}
$$

Hence:

$$
H_{i j k l}=\frac{2}{\pi} \int_{V_{\xi}} \frac{\xi_{i} \xi_{j} \xi_{k} \xi_{l}}{\xi^{4}} \exp \left(\underline{\mathbf{i}} \underline{\xi} \cdot\left(\underline{x}-\underline{x}^{\prime}\right)\right) d V_{\xi}
$$

Therefore, from Eq. A.3, $H_{i j k l}$ writes:

$$
H_{i j k l}=-2 \pi\left|\underline{x}-\underline{x}^{\prime}\right|_{, i j k l}
$$

which proves Eq. 22.

B Harmonic, bi-harmonic potential functions and Eshelby tensors for general ellipsoidal and spherical inhomogeneities

In Eq. 28, $\phi^{I}$ and $\psi^{I}$ are harmonic and bi-harmonic potential functions (Ferrers, 1877; Dyson, 1891; Eshelby, 1957) related to a general ellipsoidal inclusion $V_{I} \cdot \phi_{, i j}^{I}$ and $\psi_{, i j k l}^{I}$ are given by equation 11.35 (p. 85) in Mura (1987):

$$
\phi_{, i j}^{I}=\int_{V_{I}}\left(\frac{1}{\left|\underline{x}-\underline{x}^{\prime}\right|}\right)_{, i j} d V^{\prime} \quad \psi_{, i j k l}^{I}=\int_{V_{I}}\left|\underline{x}-\underline{x}^{\prime}\right|_{, i j k l} d V^{\prime}
$$

which are uniform when $\underline{x} \in V_{I}$. Then, the tensors $\underset{\approx}{P^{c^{M}}}$ and $\underset{\approx}{P^{b^{M}}}$ are given by:

$$
\begin{aligned}
& P_{i j k l}^{c^{M}}=-\frac{1}{8 \pi \mu^{M}}\left(\phi_{, j l}^{I} \delta_{i k}+\phi_{, i l}^{I} \delta_{j k}\right)+\frac{3 k^{M}+\mu^{M}}{8 \pi \mu^{M}\left(3 k^{M}+4 \mu^{M}\right)} \psi_{, i j k l}^{I} \\
& P_{i j k l}^{b^{M}}=-\frac{1}{8 \pi \mu_{v}{ }^{M}}\left(\phi_{, j l}^{I} \delta_{i k}+\phi_{, i l}^{I} \delta_{j k}\right)+\frac{3 k_{v}{ }^{M}+\mu_{v}{ }^{M}}{8 \pi \mu_{v}{ }^{M}\left(3 k_{v}{ }^{M}+4 \mu_{v}{ }^{M}\right)} \psi_{, i j k l}^{I}
\end{aligned}
$$

The elastic (resp. anelastic) Eshelby tensors $\underset{\approx}{\mathcal{c}^{M}}$ (resp. $\underset{\approx}{S^{b^{M}}}$ ), for elastic (resp. anelas-

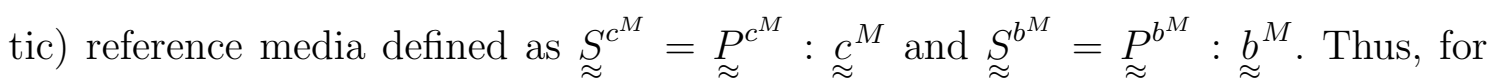


isotropic elastic and anelastic matrix phase and an arbitrary ellipsoidal inhomogeneity, $\underset{\approx}{S^{M}}$ reads:

$$
\begin{aligned}
& S_{i j m n}^{c^{M}}=-\frac{3 k^{M}-2 \mu^{M}}{4 \pi\left(3 k^{M}+4 \mu^{M}\right)} \phi_{, i j}^{I} \delta_{m n}-\frac{1}{8 \pi}\left(\phi_{, j n}^{I} \delta_{i m}+\phi_{, j m}^{I} \delta_{i n}+\phi_{, i n}^{I} \delta_{j m}+\phi_{, i m}^{I} \delta_{j n}\right) \\
& +\frac{3 k^{M}+\mu^{M}}{4 \pi\left(3 k^{M}+4 \mu^{M}\right)} \psi_{, i j m n}^{I}
\end{aligned}
$$

while $\underset{\approx}{b^{M}}$ is given by:

$$
\begin{aligned}
& S_{i j k l}^{b^{M}}=-\frac{3 k_{v}{ }^{M}-2 \mu_{v}{ }^{M}}{4 \pi\left(3 k_{v}{ }^{M}+4 \mu_{v}{ }^{M}\right)} \phi_{, i j}^{I} \delta_{m n}-\frac{1}{8 \pi}\left(\phi_{, j n}^{I} \delta_{i m}+\phi_{, j m}^{I} \delta_{i n}+\phi_{, i n}^{I} \delta_{j m}+\phi_{, i m}^{I} \delta_{j n}\right) \\
& +\frac{3 k_{v}{ }^{M}+\mu_{v}{ }^{M}}{4 \pi\left(3 k_{v}{ }^{M}+4 \mu_{v}{ }^{M}\right)} \psi_{, i j m n}^{I}
\end{aligned}
$$

For spherical inhomogeneities, the expressions of $\phi_{, i j}^{I}$ and $\psi_{, i j k l}^{I}$ (Eq. B.1) are simplified to the following expressions (Walpole, 1981; Mura, 1987):

$$
\phi_{, i j}^{I}=-\frac{4 \pi}{3} \delta_{i j} \quad \psi_{, i j k l}^{I}=-\frac{8 \pi}{15}\left(\delta_{i j} \delta_{k l}+\delta_{i k} \delta_{j l}+\delta_{i l} \delta_{j k}\right)
$$

Therefore, for spherical inhomogeneities, $\underset{\approx}{P^{c^{M}}}$ is given by:

$$
\underset{\approx}{P^{c^{M}}}=\frac{1}{3 k^{M}+4 \mu^{M}} \underset{\approx}{J}+\frac{3\left(k^{M}+2 \mu^{M}\right)}{5 \mu^{M}\left(3 k^{M}+4 \mu^{M}\right)} \underset{\approx}{K}
$$

while $\underset{\approx}{P^{M}}$ is given by:

$$
{\underset{\sim}{b^{M}}}^{b^{M}}=\frac{1}{3 k_{v}{ }^{M}+4 \mu_{v}{ }^{M}} \underset{\approx}{J}+\frac{3\left({k_{v}}^{M}+2 \mu_{v}{ }^{M}\right)}{5 \mu_{v}{ }^{M}\left(3 k_{v}{ }^{M}+4 \mu_{v}{ }^{M}\right)} \underset{\approx}{K}
$$

Besides, the time differential equation for $\underset{\sim}{\sigma^{* I}}$ is obtained from Eq. 28 together with Eq. B.6:

$$
\dot{\sigma}_{i j}^{* I}=\frac{4}{15} \alpha^{c^{M}}\left(R_{i j}^{I}-6 R_{m}^{I} \delta_{i j}\right)-\frac{\alpha^{c^{M}}}{\alpha^{b^{M}}} \sigma_{i j}^{* I}
$$




\section{Agreement with the corresponding Mori-Tanaka estimates from the correspondence principle}

The formal proof of agreement between the present Mori-Tanaka (MT) estimate for two-phase linear viscoelastic composites reported in section 3 is given by comparison with the correspondence principle by applying the Laplace-Carson (LC) transform method. For a given function $f(t)$, the Laplace-Carson transform of $f(t)$ is denoted $\widehat{f}(p)$ and is defined by

$$
\widehat{f}(p)=p \int_{0}^{\infty} f(t) e^{-p t} d t
$$

The time derivation rule is given by

$$
\widehat{\dot{f}}(p)=p(\widehat{f}(p)-f(0))
$$

In the sequel, the initial stresses and strains in the individual constituents are set to

zero, so only the part $p \widehat{f}(p)$ is considered in Eq. C.2. Using both Eqs. C.1 and C.2, the LC transform of the constitutive equation (Eq. 1) is given by

$$
\underset{\sim}{\widehat{\sigma}}(p)=\underset{\approx}{\widehat{c}}(p): \underset{\sim}{\widehat{\sim}}(p)
$$

where

$$
\underset{\approx}{\widehat{c}}(p)=\left(\underset{\approx}{s}+\frac{1}{p} \underset{\approx}{\sim}\right)^{-1}
$$

\section{C.1 Strain formulation}

The Laplace-Carson transforms of Eqs. 23 and 29 yield:

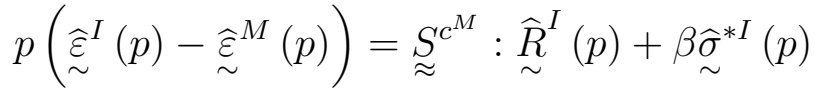

$$
\begin{aligned}
& p\left(\underset{\sim}{\widehat{\varepsilon}^{I}}(p)-{\underset{\sim}{\widehat{\varepsilon}}}^{M}(p)\right)=\underset{\approx}{S^{b^{M}}}: \underset{\sim}{\widehat{R}}{ }^{I}(p)+p \gamma \underset{\sim}{\widehat{\sigma}^{* I}}(p)
\end{aligned}
$$


Combining both equations in Eq. C.5 to eliminate $\underset{\sim}{\widehat{\sigma}^{* I}}(p)$ yields:

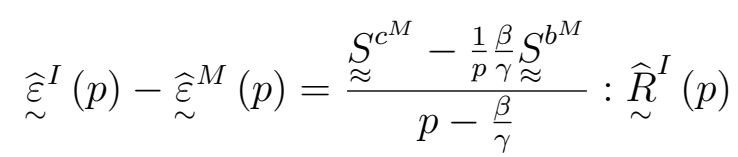

In the present formulation, the matrix phase properties $\underset{\approx}{c^{M}}$ and $\underset{\approx}{m^{M}}$ are isotropic, see section 2. Thus, the "elastic symbolic" behavior of the matrix phase is given by:

$$
{\underset{\approx}{\widehat{c}^{M}}}^{M}(p)=3 \widehat{k}^{M}(p) \underset{\approx}{J}+2 \widehat{\mu}^{M}(p) \underset{\approx}{K}
$$

where:

$$
\begin{aligned}
\widehat{k}^{M}(p) & =\frac{k^{M} p}{p+\frac{k^{M}}{k_{v}}} \\
\widehat{\mu}^{M}(p) & =\frac{\mu^{M} p}{p+\frac{\mu^{M}}{\mu_{v}}}
\end{aligned}
$$

For the inhomogeneity phase, $\underset{\underset{\widetilde{c}}{I}}{\widehat{c}^{I}}(p)$ is anisotropic, see Eq. C.4. Thus, the expression of $p-\frac{\beta}{\gamma}$ in Eq. C.6 is straightforward from Eqs. 24, 30 and C.8:

$$
p-\frac{\beta}{\gamma}=p \frac{k^{M} \mu^{M}}{\widehat{k}^{M}(p) \widehat{\mu}^{M}(p)} \frac{3 \widehat{k}^{M}(p)+4 \widehat{\mu}^{M}(p)}{3 k^{M}+4 \mu^{M}}
$$

and the expression of $\underset{\approx}{S^{c^{M}}}-\frac{1}{p} \frac{\beta}{\gamma}{\underset{\approx}{b^{M}}}^{b^{M}}$ in Eq. C.6 is obtained from Eq. C.8 together with Eqs. B.4 and B.5:

$$
\begin{aligned}
& S_{i j m n}^{c^{M}}-\frac{1}{p} \frac{\beta}{\gamma} S_{i j m n}^{b^{M}}=-\frac{1}{4 \pi}\left(\frac{k^{M} \mu^{M}}{\widehat{k}^{M}(p) \widehat{\mu}^{M}(p)} \frac{3 \widehat{k}^{M}(p)-2 \widehat{\mu}^{M}(p)}{3 k^{M}+4 \mu^{M}}\right) \phi_{, i j}^{I} \delta_{m n} \\
& -\frac{1}{8 \pi}\left(\frac{k^{M} \mu^{M}}{\widehat{k}^{M}(p) \widehat{\mu}^{M}(p)} \frac{3 \widehat{k}^{M}(p)+4 \widehat{\mu}^{M}(p)}{3 k^{M}+4 \mu^{M}}\right)\left(\phi_{, j n}^{I} \delta_{i m}+\phi_{, j m}^{I} \delta_{i n}+\phi_{, i n}^{I} \delta_{j m}+\phi_{, i m}^{I} \delta_{j n}\right) \\
& +\frac{1}{4 \pi}\left(\frac{k^{M} \mu^{M}}{\widehat{k}^{M}(p) \widehat{\mu}^{M}(p)} \frac{3 \widehat{k}^{M}(p)+\widehat{\mu}^{M}(p)}{3 k^{M}+4 \mu^{M}}\right) \psi_{, i j m n}^{I}
\end{aligned}
$$

Thus, introducing Eqs. C.9 and C.10 within Eq. C.6 leads to:

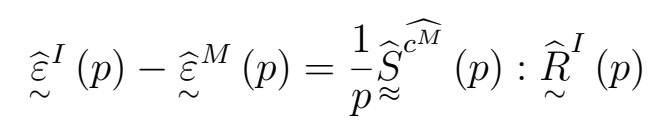

where $\underset{\approx}{\widehat{S}^{\widehat{M}}}(p)$ is the "symbolic" counterpart of $\underset{\approx}{{ }^{c^{M}}}$ (Eq. B.4) associated with the 
"elastic symbolic" property of the matrix phase $\underset{\approx}{\widehat{c}^{M}}(p)$ (Eq. C.7):

$$
\begin{aligned}
& \widehat{S}_{i j m n}^{\widehat{c M}}(p)=-\frac{3 \widehat{k}^{M}(p)-2 \widehat{\mu}^{M}(p)}{4 \pi\left(3 \widehat{k}^{M}(p)+4 \widehat{\mu}^{M}(p)\right)} \phi_{, i j}^{I} \delta_{m n}-\frac{1}{8 \pi}\left(\phi_{, j n}^{I} \delta_{i m}+\phi_{, j m}^{I} \delta_{i n}+\phi_{, i n}^{I} \delta_{j m}+\phi_{, i m}^{I} \delta_{j n}\right) \\
& +\frac{3 \widehat{k}^{M}(p)+\widehat{\mu}^{M}(p)}{4 \pi\left(3 \widehat{k}^{M}(p)+4 \widehat{\mu}^{M}(p)\right)} \psi_{, i j m n}^{I}
\end{aligned}
$$

In Eq. C.11, $\underset{\sim}{\stackrel{R}{R}}(p)$ is obtained by transforming Eq. 25 using Eq. C.1:

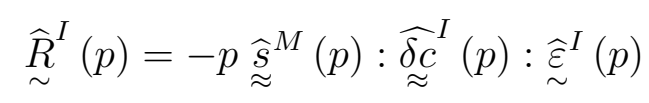

Introducing Eq. C.13 in Eq. C.11 gives the relationship between $\underset{\sim}{\widehat{\varepsilon}^{I}}(p)$ and $\underset{\sim}{\widehat{\varepsilon}^{M}}(p)$ :

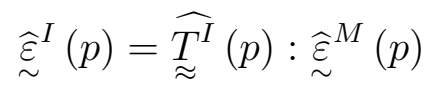

where $\underset{\approx}{\widehat{T}}(p)$ is given by:

$$
\widehat{\widetilde{T}}^{I}(p)=\left(\underset{\approx}{I}+\underset{\approx}{\widehat{\sim}^{c^{M}}}(p): \underset{\approx c}{\widehat{\delta c}^{I}}(p)\right)^{-1}
$$

with:

$$
\widehat{P}_{i j k l}^{c^{M}}(p)=-\frac{1}{8 \pi \widehat{\mu}^{M}(p)}\left(\phi_{, j l}^{I} \delta_{i k}+\phi_{, i l}^{I} \delta_{j k}\right)+\frac{3 \widehat{k}^{M}(p)+\widehat{\mu}^{M}(p)}{8 \pi \widehat{\mu}^{M}(p)\left(3 \widehat{k}^{M}(p)+4 \widehat{\mu}^{M}(p)\right)} \psi_{, i j k l}^{I}
$$

The LC transform of Eq. 33 in section 3 to find the MT estimate yields:

$$
\underset{\sim}{\widehat{E}}(p)=f \underset{\sim}{\widehat{\varepsilon}^{I}}(p)+(1-f) \underset{\sim}{\widehat{\varepsilon}^{M}}(p)
$$

Therefore, using Eq. C.14 together with Eq. C.17, the strain concentration equation for $\underset{\sim}{\widehat{\varepsilon}^{I}}(p)$ with the MT estimate (Benveniste, 1987) using the correspondence principle is retrieved:

$$
\underset{\sim}{\widehat{\varepsilon}^{I}}(p)=\underset{\approx}{\widehat{A^{I}}}(p): \underset{\sim}{\widehat{E}}(p)
$$


where $\widehat{A}^{I}(p)$ is the "elastic symbolic" strain concentration tensor of the inhomogeneity phase $I$ :

$$
{\underset{\approx}{A}}^{\widehat{A}}(p)=\left(\underset{\approx}{I}+(1-f) \underset{\approx}{\underset{\approx}{{ }^{\widehat{M}}}}(p): \underset{\approx c}{\widehat{\delta c}^{I}}(p)\right)^{-1}
$$

or, after simple algebraic manipulations using Eq. C.15:

$$
\widehat{\approx}^{\widehat{A}^{I}}(p)=\widehat{\approx}^{\widehat{T}}(p):\left((1-f) \underset{\approx}{I}+f \widehat{\approx}^{\underline{T}}(p)\right)^{-1}
$$

From the definition of $\underset{\sim}{R^{I}}$ (Eq. 25), another expression is:

$$
\underset{\sim}{R}=\frac{1}{f}\left(\underset{\sim}{\dot{E}}-\underset{\approx}{\underset{\approx}{s}}: \underset{\sim}{\dot{\Sigma}}-\underset{\approx}{m^{M}}: \underset{\sim}{\Sigma}\right)
$$

Then, using Eq. C.1, the LC transform of $\underset{\sim}{R^{I}}$ (Eq. C.21) reads:

$$
\underset{\sim}{\widehat{R^{I}}}(p)=\frac{p}{f}(\underset{\sim}{\widehat{E}}(p)-\underset{\approx}{\widehat{\widetilde{s}}}(p): \underset{\sim}{\widehat{\Sigma}}(p))
$$

Therefore, using Eq. C.11 together with Eq. C.22 yields:

$$
\underset{\sim}{\widehat{\mathcal{\varepsilon}}^{I}}(p)={\underset{\sim}{\widehat{\varepsilon}^{M}}}^{M}(p)+\frac{1}{f} \underset{\widetilde{\sim}}{\widehat{c}^{c^{M}}}(p):\left(\underset{\sim}{\widehat{E}}(p)-{\underset{\approx}{\widehat{s}}}^{\widehat{S}}(p): \underset{\sim}{\widehat{\Sigma}}(p)\right)
$$

Introducing Eqs. C.14 and C.18 into Eq. C.23, it is found that the effective "elastic symbolic" behavior of the two-phase composite agrees with the one given by the correspondence principle using the MT estimate (Weng, 1984; Benveniste, 1987), see e.g. Wang and Weng (1992):

$$
\widehat{\sim}(p)=\underset{\sim}{c^{e f f}}(p): \underset{\sim}{\widehat{E}}(p)
$$

with the effective "elastic symbolic" stiffness tensor $\underset{\approx}{\overbrace{}^{e f f}}(p)$ :

$$
\underset{\approx}{\widehat{c^{e f f}}}(p)=\underset{\approx}{\widehat{c^{M}}}(p)+f\left(\underset{\approx}{\widehat{c^{I}}}(p)-\underset{\approx}{\widehat{c^{M}}}(p)\right): \underset{\approx}{\widehat{A^{I}}}(p)
$$




\section{C.2 Stress formulation}

The Laplace-Carson transform of Eqs. 27 and 32 yield:

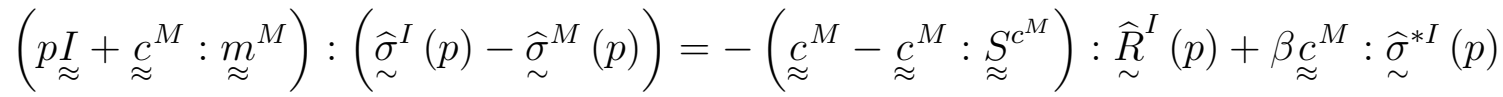

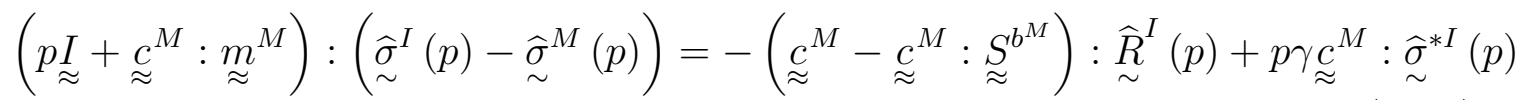

Combining both equations in Eq. C.26 to eliminate $\widehat{\sigma}_{\sim}^{* I}(p)$ yields:

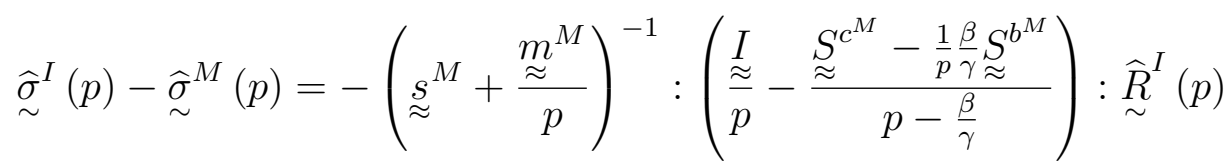

Then, using Eqs. C.9 and C.10:

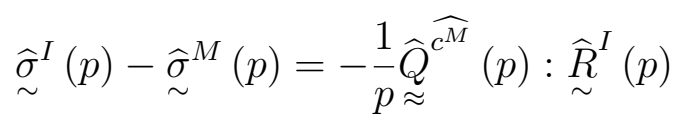

where $\underset{\underset{Q}{\widehat{Q}^{M}}}{\widehat{c^{M}}}(p)=\underset{\approx}{\widehat{c^{M}}}(p)-{\underset{\approx}{\widehat{c} M}}^{\widehat{\approx}}(p): \widehat{\approx}^{\widehat{c^{M}}}(p)$, and, $\underset{\sim}{\widehat{R}}(p)$ is given by the LC transform of Eq. 25:

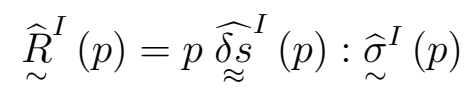

Introducing Eq. C.29 in Eq. C.28 gives the relationship between $\underset{\sim}{\widehat{\sigma}^{I}}(p)$ and $\underset{\sim}{\widehat{\sigma}^{M}}(p)$ :

$$
{\underset{\sim}{\widehat{\sigma}}}^{I}(p)=\left(\underset{\approx}{I}+\underset{\approx}{\widehat{\widetilde{Q}}} \widehat{c}^{c^{M}}(p): \underset{\approx}{\widehat{\delta s}^{I}}(p)\right)^{-1}:{\underset{\sim}{\widehat{\sigma}}}^{M}(p)
$$

The LC transform of Eq. 38 in section 3 to find the MT estimate yields:

$$
\underset{\sim}{\widehat{\Sigma}}(p)=f \underset{\sim}{\widehat{\sigma}^{I}}(p)+(1-f) \underset{\sim}{\widehat{\sigma}^{M}}(p)
$$

Then, using Eq. C.30 together with Eq. C.31, the stress concentration equation for $\underset{\sim}{\widehat{\varepsilon}^{I}}(p)$ with the MT estimate (Benveniste, 1987) using the correspondence principle 
is retrieved:

$$
\widehat{\sim}^{I}(p)=\underset{\approx}{\widehat{B^{I}}}(p): \underset{\sim}{\widehat{\Sigma}}(p)
$$

where $\underset{B^{I}}{\approx}(p)$ is the "elastic symbolic" stress concentration tensor of the inhomogeneity phase $I$ :

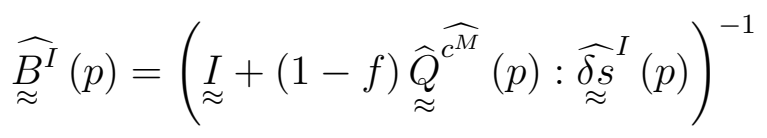

or, after simple algebraic manipulations using tensor $\underset{\approx}{\widehat{T}^{I}}(p)$ defined in Eq. C.15:

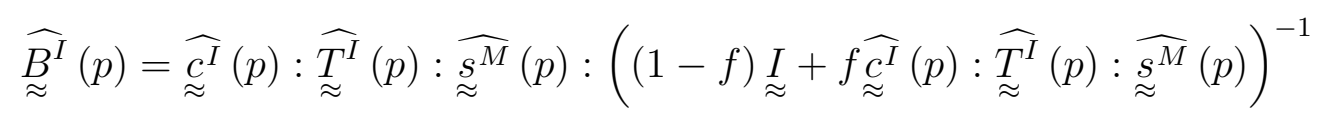

Besides, using Eq. C.22 in Eq. C.28 yields:

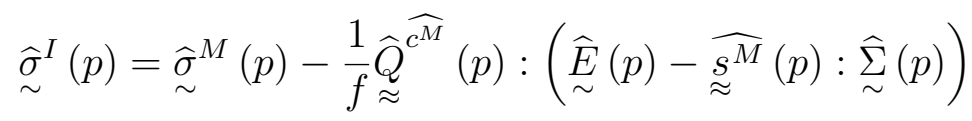

Introducing Eqs. C.30 and C.32 into Eq. C.35, it is found that the effective "elastic symbolic " behavior of the two-phase composite agrees with the one given by the correspondence principle using the MT estimate (Weng, 1984; Benveniste, 1987), see e.g. Wang and Weng (1992):

$$
\underset{\sim}{\widehat{E}}(p)=\underset{\approx}{\widehat{乛}} \widehat{\widehat{e f f}}(p): \underset{\sim}{\widehat{\Sigma}}(p)
$$

with the effective "elastic symbolic" compliance tensor $\underset{\approx}{\widehat{\text { eff }}}(p)$ :

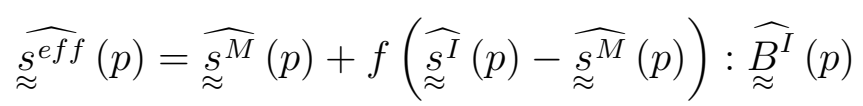

\section{References}

Agoras, M., Avazmohammadi, R., Ponte Castañeda, P., 2016. Incremental variational procedure for elasto-viscoplastic composites and application to polymer and metal matrix composites reinforced by spheroidal elastic particles. Int. J. Solids Struct. 97-98, 668-686. 
Badulescu, C., Lahellec, N., Suquet, P., ., 2015. Field statistics in linear viscoelastic composites and polycrystals. Eur. J. Mech. A. Solids 49, 329-344.

Benveniste, Y., 1987. A new approach to the application of Mori-Tanaka's theory in composite materials. Mech. Mater. 6 (2), 147-157.

Berbenni, S., Capolungo, L., 2015. A Mori-Tanaka homogenization scheme for nonlinear elasto-viscoplastic heterogeneous materials based on translated fields: An affine extension. C. R. Mecanique 343, 95-106.

Berbenni, S., Dinzart, F., Sabar, H., 2015. A new internal variables homogenization scheme for linear viscoelastic materials based on an exact Eshelby interaction law. Mech. Mater. 81, 110-124.

Berbenni, S., Sabar, H., 2017. A time-incremental Eshelby-based homogenization scheme for viscoelastic heterogeneous materials (chapter 12). In: Micromechanics and Nanomechanics of Composite Solids (Editors: Shaker A. Meguid, George J. Weng). ISBN 978-3-319-52793-2, Springer, pp. 347-369.

Brassard, L., Stainier, L., Doghri, I., Delannay, L., 2012. Homogenization of elasto(visco)plastic composites based on an incremental variational principle. Int. J. Plast. 36, 86-112.

Brinson, L. C., Lin, W. S., 1998. Comparison of micromechanics methods for effective properties of multiphase viscoelastic composites. Compos. Struct. 41, 353-367.

Cotelo, J., Das, S., Ponte Castañeda, P., 2020. A differential homogenization method for estimating the macroscopic response and field statistics of particulate viscoelastic composites. Int. J. Solids Struct. 204-205, 199-219.

Czarnota, C., Kowalczyk-Gajewska, K., Salahouelhadj, A., Martiny, M., Mercier, S., 2015. Modeling of the cyclic behavior of elastic-viscoplastic composites by the additive tangent mori-tanaka approach and validation by finite element calculations. Int. J. Solids Struct. 56-57, 96-117.

Das, S., Ponte Castañeda, P., 2021. Differential variational estimates for the macro- 
scopic response and field statistics of elasto-viscoplastic polycrystals. J. Mech. Phys. Solids 147, 104202.

DeBotton, G., Tevet-Deree, L., 2004. The response of a fiber-reinforced composite with a viscoelastic matrix phase. J. Compos. Mater. 38, 1255-1277.

Doghri, I., Adam, L., Bilger, N., 2010. Mean-field homogenization of elastoviscoplastic composites based on a general incrementally affine linearization method. Int. J. Plast. 26, 219-238.

Dyson, F., 1891. The potentials of ellipsoids of variable densities. Q. J. Pure Appl. Math. 25, 259-288.

Eshelby, J. D., 1957. The determination of the elastic field of an ellipsoidal inclusion and related problems. Proc. Roy. Soc. London A. 241, 376-396.

Eshelby, J. D., 1961. Elastic inclusions and inhomogeneities). In: Progress in Solid Mecahnics, Vol. II (Editors: I.N. Sneddon, R. Hill). North Holland Publishing Company, Amsterdam, pp. 89-140.

Ferrers, N., 1877. On the potentials of ellipsoids, ellipsoidal shells, elliptic laminae and elliptic rings of variable densities. Q. J. Pure Appl. Math. 14, 1-22.

Hashin, Z., 1969. The inelastic inclusion problem. Int. J. Eng. Sci. 7, 11-36.

Hill, R., 1967. The essential structure of constitutive laws for metal composites and polycrystals. J. Mech. Phys. Solids 15, 79-95.

Hu, G. K., 1996. A method of plasticity for general aligned spheroidal void or fiberreinforced composites. Int. J. Plast. 12, 439-449.

Kouddane, R., Molinari, A., Canova, G. R., 1993. Self-consistent modelling of heterogeneous viscoelastic and elastoplastic materials. In: Mecamat 91: Large Plastic Deformations, Fundamentals and Application to Metal Forming. C. Teodosiu, J.L. Raphanel, F. Sidoroff (Eds), Balkema,Rotterdam, pp. 129-141.

Kowalczyk-Gajewska, K., Petryk, H., 2011. Sequential linearization method for viscous/elastic heterogeneous materials. Eur. J. Mech. A Solids 30, 650-664. 
Kröner, E., 1990. Modified Green functions in the theory of heterogeneous and/or anisotropic linearly elastic media. In: Micromechanics and Inhomogeneity. The Toshio Mura 65th Anniversary Volume. G.J. Weng, M. Taya, H. Abe (eds.), Springer Verlag, New York, pp. 197-211.

Lahellec, N., Suquet, P., 2007a. Effective behavior of linear viscoelastic composites: A time-integration approach. Int. J. Solids Struct. 44, 507-529.

Lahellec, N., Suquet, P., 2007b. On the effective behavior of non linear inelastic composites: I. Incremental variational principles. J. Mech. Phys. Solids 55, 19321963.

Lahellec, N., Suquet, P., 2013. Effective response and field statistics in elasto-plastic and elasto-viscoplastic composites under radial and non radial loadings. Int. J. Plast. 42, 1-30.

Laws, N., McLaughlin, R., 1978. Self-consistent estimates for the viscoelastic creep compliance of composite materials. Proc. Roy. Soc. London A 359, 251-273.

Lhadi, S., Berbenni, S., Gey, N., Richeton, T., Germain, L., 2018. Micromechanical modeling of the effect of elastic and plastic anisotropies on the mechanical behavior of beta-Ti alloys. Int. J. Plast. 109, 88-107.

Li, J., Weng, G. J., 1994. Strain-rate sensitivity, relaxation behavior and complex moduli of a class of isotropic viscoplastic composites. ASME J. Eng. Mater. Tech. $116,495-504$.

Li, J., Weng, G. J., 1997. A secant-viscosity approach to the time-dependent creep of an elastic-viscoplastic composite. J. Mech. Phys. Solids 45 (7), 1069-1083.

Mandel, J., 1966. Cours de Mécanique des Milieux Continus. Gauthiers-Villars, Paris.

Mareau, C., Berbenni, S., 2015. An affine formulation for the self-consistent modeling of elasto-viscoplastic heterogeneous materials based on the translated field method. Int. J. Plast. 64, 134-150. 
Masson, R., Brenner, R., Castelnau, O., 2012. Incremental homogenization approach for ageing viscoelastic polycrystals. C. R. Mecanique 340, 378-386.

Masson, R., Seck, M. E. B., Fauque, J., Garajeu, M., 2020. Homogenization schemes for aging linear viscoelastic matrix-inclusion composite materials with elongated inclusions. J. Mech. Phys. Solids 137, 103874.

Masson, R., Zaoui, A., 1999. Self-consistent estimates for the rate-dependent elastoplastic behaviour of polycrystalline materials. J. Mech. Phys. Solids 47, 1543-1568.

Mercier, S., Jacques, N., Molinari, A., 2005. Validation of an interaction law for the eshelby inclusion problem in elasto-viscoplasticity. Int. J. Solids Struct. 1223-1241, 42.

Mercier, S., Molinari, A., 2009. Homogenization of elasticviscoplastic heterogeneous materials: self-consistent and moritanaka schemes. Int. J. Plast. 25, 1024-1048.

Mercier, S., Molinari, A., Berbenni, S., Berveiller, M., 2012. Comparison of different homogenization approaches for elastic-viscoplastic materials. Modell. Simul. Mater. Sci. Eng. 20, 024004.

Molinari, A., 2002. Averaging models for heterogeneous viscoplastic and elasticviscoplastic materials. ASME J. Eng. Mater. Tech. 124, 62-70.

Molinari, A., Ahzi, S., Kouddane, R., 1997. On the self-consistent modelling of elastic-plastic behavior of polycrystals. Mech. Mater. 26, 43-62.

Mori, T., Tanaka, K., 1973. Average stress in matrix and average elastic energy of materials with misfitting inclusions. Acta Metall. 21, 571-574.

Mura, T., 1987. Micromechanics of defects in solids. Kluwer Academic Publishers, Dordrecht, The Netherlands.

Paquin, A., Sabar, H., Berveiller, M., 1999. Integral formulation and self-consistent modelling of elasto-viscoplastic behavior of heterogeneous materials. Arch. Appl. Mech. 69, 14-35.

Pierard, O., Doghri, I., 2006. An enhanced affine formulation and the correspond- 
ing numerical algorithms for the mean-field homogenization of elasto-viscoplastic composites. Int. J. Plast. 22, 131-157.

Ponte Castañeda, P., 1991. The effective mechanical properties of nonlinear isotropic composites. J. Mech. Phys. Solids 39, 45-71.

Ponte Castañeda, P., Suquet, P., 1998. Nonlinear composites. Adv. Appl. Mech. 34, $171-302$.

Qiu, Y. P., Weng, G. J., 1991. A theory of plasticity for porous materials and particlereinforced composites. ASME J. Appl. Mech. 59, 261-268.

Ricaud, J. M., Masson, R., 2009. Effective properties of linear viscoelastic heterogeneous media: internal variables formulation and extension to ageing behaviours. Int. J. Solids Struct. 46, 1599-1606.

Rougier, Y., Stolz, C., Zaoui, A., 1993. Representation spectrale en viscoelasticite lineaire des materiaux heterogenes. C. R. Acad. Sci. Paris Ser. II 316, 1517-1522.

Rougier, Y., Stolz, C., Zaoui, A., 1994. Self-consistent modelling of elasticviscoplastic polycrystals. C. R. Acad. Sci. Paris Ser. IIb 318, 145-151.

Sabar, H., Berveiller, M., Favier, V., Berbenni, S., 2002. A new class of micro-macro models for elastic-viscoplastic heterogeneous materials. Int. J. Solids Struct. 39, $3257-3276$

Suquet, P., 1987. Elements of homogenization for inelastic solid mechanics. In: Homogenization techniques for Composite media. E. Sanchez-Palencia and A. Zaoui (Eds), Springer Berlin, pp. 193-278.

Suquet, P., 1995. Overall properties of nonlinear composites: a modified secant moduli theory and its link with Ponte Castañedas nonlinear variational procedure. C. R. Acad. Sci. Paris Serie IIb 320, 563571.

Turner, P. A., Tomé, C. N., 1993. Self-consistent modeling of visco-elastic polycrystals: Application to irradiation creep and growth. J. Mech. Phys. Solids 41 (7), $1191-1211$ 
Vu, Q. H., Brenner, R., Castelnau, O., Moulinec, H., Suquet, P., 2005. A selfconsistent estimate for linear viscoelastic polycrystals with internal variables inferred from the collocation method. Modell. Simul. Mater. Sci. Eng. 20, 024003.

Walpole, L. J., 1966. On bounds for the overall elastic moduli of inhomogeneous systems. i. J. Mech. Phys. Solids 14, 151-162.

Walpole, L. J., 1981. Elastic behavior of composite materials: theoretical foundations. Adv. Appl. Mech. 21, 169-242.

Wang, H., Wu, P. D., Tomé, C. N., Huang, Y., 2010. A finite strain elasticviscoplastic self-consistent model for polycrystalline materials. J. Mech. Phys. Solids 58, 594-612.

Wang, Y. M., Weng, G. J., 1992. The influence of inclusion shape on the overall viscoelastic behavior of composites. ASME J. Appl. Mech. 59 (3), 510-518.

Weng, G. J., 1984. Some elastic properties of reinforced solids with special reference to isotropic ones containing spherical inclusions. Int. J. Eng. Sci. 22 (7), 845-856.

Weng, G. J., 1993. A self-consistent relation for the time-dependent creep of polycrystals. Int. J. Plast. 9, 181-198.

Wu, L., Adam, L., Doghri, I., Noels, L., 2017. An incremental-secant mean field homogenization method with second statistical moments for elasto-visco-plastic composite materials. Mech. Mater. 114, 180-200.

Zecevic, M., Lebensohn, R. A., 2020. New robust self-consistent schemes for elasticviscoplastic polycrystals. Int. J. Solids Struct. 202, 434-453. 